\title{
On the correction to Einstein's formula for the effective viscosity
}

\author{
David Gérard-Varet and Amina Mecherbet
}

\begin{abstract}
This paper is a follow-up to Gérard-Varet and Hillairet (2020) on the derivation of accurate effective models for viscous dilute suspensions. The goal is to identify an effective Stokes equation providing an $o\left(\lambda^{2}\right)$ approximation of the exact fluid-particle system, with $\lambda$ the solid volume fraction of the particles. This means that we look for an improvement of Einstein's formula for the effective viscosity in the form $\mu_{\text {eff }}(x)=\mu+\frac{5}{2} \mu \rho(x) \lambda+\mu_{2}(x) \lambda^{2}$. Under a separation assumption on the particles, we proved in the article above that if an $o\left(\lambda^{2}\right)$ Stokes effective approximation exists, the correction $\mu_{2}$ is necessarily given by a mean field limit, which can then be studied and computed under further assumptions on the particle configurations. Roughly, we go here from the conditional result of the article above to an unconditional result: we show that such an $o\left(\lambda^{2}\right)$ Stokes approximation indeed exists, as soon as the mean field limit exists. This includes the case of periodic and random stationary particle configurations.
\end{abstract}

\section{Introduction}

We consider a suspension of spherical particles, modelled by a collection of balls $B_{i}=$ $B\left(x_{i}, r_{n}\right), 1 \leq i \leq n$, all included in a fixed compact of $\mathbb{R}^{3}$. The centres $x_{i}$ of the balls may (and will) depend on $n$, but we omit it in the notation. We consider a regime where $n$ is large, and $r_{n} \sim n^{-1 / 3}$. More precisely, we assume for simplicity that $\lambda:=n \frac{4}{3} \pi r_{n}^{3}$ is independent of $n$. We also assume that the balls occupy a volume of size 1 , in the sense that

$$
\rho_{n}:=\frac{1}{n} \sum_{i=1}^{n} \delta_{x_{i}} \rightarrow \rho(x) d x, \quad n \rightarrow+\infty,
$$

where $\rho$ is a bounded density with support $\overline{\mathcal{O}}$ for a smooth bounded domain $\mathcal{O}$ such that $|\mathcal{O}|=1$. In particular, $\lambda$ can be interpreted as the solid volume fraction. The suspension is immersed in a viscous fluid. We consider particles light enough so that neglecting inertia of the fluid and the particles is reasonable. Setting $\Omega_{n}:=\mathbb{R}^{3} \backslash \bigcup_{i} B_{i}$,

$$
\begin{cases}-\operatorname{div}\left(2 \mu D\left(u_{n}\right)-\mathbb{I} p_{n}\right)=g_{n} & \text { on } \Omega_{n}, \\ \operatorname{div}\left(u_{n}\right)=0 & \text { on } \Omega_{n}, \\ u_{n}=u_{i}+\omega_{i} \times\left(x-x_{i}\right) & \text { on } B_{i},\end{cases}
$$


where $g_{n} \in L^{2}\left(\mathbb{R}^{3}\right) \cap L^{6 / 5}\left(\mathbb{R}^{3}\right)$ models some forcing. The constant vectors $u_{i}$ and $\omega_{i}$ are the translation velocity and rotation vector of ball $B_{i}$. They are unknowns, associated to the Newtonian dynamics of the particles: in the absence of inertia, relations are of the form

$$
\begin{aligned}
\int_{\partial B_{i}} \sigma\left(u_{n}, p_{n}\right) v & =-\int_{B_{i}} g_{n} d x \\
\int_{\partial B_{i}}\left[\sigma\left(u_{n}, p_{n}\right) v\right] \times\left(x-x_{i}\right) & =-\int_{B_{i}} g_{n} \times\left(x-x_{i}\right) d x,
\end{aligned}
$$

where $\sigma(u, p)=2 D(u)-p I$ is the Newtonian stress tensor, and $v$ is the unit normal vector pointing outward. These relations correspond to prescribing the force and the torque on each particle. One further assumes decay of $u_{n}$ at infinity, which will be encoded in the functional setting.

As $n \rightarrow+\infty$, one may expect that some averaging takes place. The hope is to replace the fluid-particle system above by a Stokes equation, with a viscosity coefficient $\mu_{\mathrm{eff}}=$ $\mu_{\text {eff }}(x)$, different from $\mu$ in the domain $\mathcal{O}$, reflecting there the rigidity of the particles. Convergence to such a Stokes equation can indeed be shown through homogenization techniques, if one further assumes periodicity or stationarity assumptions on the distributions of balls: see [4], or [13] for the scalar case. Note that such homogenization results are valid for any $\lambda$, but somehow abstract, as the expression of the effective viscosity involves a corrector equation which is not much simpler than the original system. They are, moreover, restricted to homogeneous distributions. In the present paper, we aim at more explicit formulas for the effective viscosity in the dilute regime, namely when $\lambda$ is small (but not vanishing as $n$ goes to infinity). We want to show that for $n$ large, the solution $u_{n}$ of (1) has for $o\left(\lambda^{2}\right)$ approximation the solution $\bar{u}$ of

$$
\begin{cases}-\operatorname{div}\left(2\left[\mu+\mu_{1} \lambda+\mu_{2} \lambda^{2}\right] D(\bar{u})-\mathbb{I} \bar{p}\right)=g & \text { on } \mathbb{R}^{3}, \\ \operatorname{div}(\bar{u})=0 & \text { on } \mathbb{R}^{3},\end{cases}
$$

for appropriate first- and second-order corrections $\mu_{1}=\mu_{1}(x), \mu_{2}=\mu_{2}(x)$. Clearly, $\mu_{1}$ and $\mu_{2}$ should be nonzero only in the region $\mathcal{O}$ where the suspension is located. Note also that, if the distribution of the particles is anisotropic, $\mu_{1}$ and $\mu_{2}$ are not expected to be scalar functions. In full generality, we look for $\mu_{1}, \mu_{2}$ in the set

$$
\operatorname{Sym}\left(\operatorname{Sym}_{3, \sigma}(\mathbb{R})\right):=\left\{M: \operatorname{Sym}_{3, \sigma}(\mathbb{R}) \rightarrow \operatorname{Sym}_{3, \sigma}(\mathbb{R}), M^{t}=M\right\}
$$

of symmetric isomorphisms of the space of trace-free symmetric $3 \times 3$ matrices (denoted by $\left.\operatorname{Sym}_{3, \sigma}(\mathbb{R})\right)$. This space can be identified with the space of four-tensors satisfying

$$
M=\left(M_{i j k l}\right)_{1 \leq i, j, k, l \leq 3}, \quad M_{i j k l}=M_{j i k l}=M_{j i l k}=M_{l k j i},
$$

and the trace conditions

$$
\sum_{i} M_{i i k l}=0 \text { for } k \neq l, \quad \sum_{i} M_{i i 11}=\sum_{i} M_{i i 22}=\sum_{i} M_{i i 33} .
$$


The search for the effective viscosity has a long history, starting from the work of Einstein ([5]): he showed that if the suspension is homogeneous, and if the interaction between the particles can be neglected, an $o(\lambda)$ approximation is given by $\mu_{\text {eff }}=\mu+$ $\frac{5}{2} \lambda \mu$. A rigorous derivation of this formula and of inhomogeneous extensions was later provided under more or less stringent separation assumptions on the particles. We refer to $[10,14,16]$ for periodic distributions of balls, and to $[12,15]$ where the periodicity assumption is relaxed into a lower bound on the minimal distance:

$$
d_{n}:=\min _{i \neq j}\left|x_{i}-x_{j}\right| \geq c n^{-1 / 3} .
$$

See also the most recent paper [9], where the formula $\mu_{1}(x)=\frac{5}{2} \rho(x) \mu$ is established under mild requirements. Our concern in the present paper is related to an $o\left(\lambda^{2}\right)$ effective approximation that is beyond Einstein's formula. Such second-order effective viscosity has been discussed in several papers; see $[1,2,19]$. However, one can observe discrepancies between the results, and very different approaches depending on the types of suspensions considered. A more global analysis was initiated by the first author and M. Hillairet in the recent paper [8]. Roughly, this paper shows that under (A1), if $\lim \sup _{n}\left\|u_{n}-\bar{u}\right\|=o\left(\lambda^{2}\right)$, where \|\| is a weak norm and where $\bar{u}$ is a solution of (3) with $\mu_{1}=\frac{5}{2} \rho(x) \mu$, then necessarily,

$$
v_{2}:=\int_{\mathcal{O}} \mu_{2}=\frac{75}{16 \pi} \lim _{n \rightarrow \infty}\left(\frac{1}{n^{2}} \sum_{j \neq i} \mathcal{M}\left(x_{i}-x_{j}\right)-\iint \mathcal{M}(x-y) \rho(x) \rho(y) d x d y\right)
$$

where $\mathcal{M}=\mathcal{M}(x) \in \operatorname{Sym}\left(\operatorname{Sym}_{3, \sigma}(\mathbb{R})\right)$ is given by

$$
\begin{aligned}
\mathcal{M}(x) S: S^{\prime} & =-D\left(\frac{x \otimes x: S}{|x|^{5}} x\right): S^{\prime} \\
& =-2 \frac{S x \cdot S^{\prime} x}{|x|^{5}}+5 \frac{(S: x \otimes x)\left(S^{\prime}: x \otimes x\right)}{|x|^{7}} \quad \forall S, S^{\prime} \in \operatorname{Sym}_{3, \sigma}(\mathbb{R}) .
\end{aligned}
$$

Hence, if a second-order effective model exists, the average $v_{2}$ of the second-order correction over the domain (which coincides with $\mu_{2}$ if $\mu_{2}$ does not depend on $x$ ) is given by the mean field limit (4)-(5). The second part of article [8] consists of an analysis of such a mean field limit, using ideas introduced by S. Serfaty and her coauthors in the context of Coulomb gases (see [17] and references therein). More explicit formulas are provided, notably in the periodic case.

The limitation of the results in [8] is that they hold conditionally to the existence of a second-order effective model of type (3). Ideally, one would like to prove the existence of an effective model as soon as the mean field limit in (4)-(5) does exist. This necessary condition is however not enough: indeed, $v_{2}$ corresponds to an average over the whole domain $\mathcal{O}$, so that it is unlikely to guarantee the existence of an effective local coefficient $\mu_{2}=\mu_{2}(x)$. Nevertheless, as we will show, we can exhibit more local in nature mean field limits, whose existence ensures the existence of an effective model. Moreover, such 
limits allow us to determine $\mu_{2}$, and not only its average $v_{2}$. We introduce

$$
\mu_{2, n}:=\frac{75 \mu}{16 \pi}\left(\mathcal{M}(x-y) 1_{x \neq y} \rho_{n}(d x) \rho_{n}(d y)-\mathcal{M}(x-y) \rho(x) d x \rho(y) d y\right) .
$$

It can be seen as a compactly supported distribution on $\mathbb{R}_{x}^{3} \times \mathbb{R}_{y}^{3}$, with values in the space $\operatorname{Sym}\left(\operatorname{Sym}_{3, \sigma}(\mathbb{R})\right)$ : for $F=F(x, y) \in C^{\infty}\left(\mathbb{R}^{3} \times \mathbb{R}^{3}\right)\left(\right.$ even for $\left.F \in C^{1}\left(\mathbb{R}^{3} \times \mathbb{R}^{3}\right)\right)$,

$$
\begin{aligned}
\left\langle\mu_{2, n}, F\right\rangle=\frac{75 \mu}{16 \pi}( & \frac{1}{n^{2}} \sum_{i \neq j} \mathcal{M}\left(x_{i}-x_{j}\right) F\left(x_{i}, x_{j}\right) \\
& \left.-\int_{\mathbb{R}^{3}} \int_{\mathbb{R}^{3}} \mathcal{M}(x-y) F(x, y) \rho(x) \rho(y) d y d x\right) .
\end{aligned}
$$

We stress that $\mathcal{M}(x)$ is a Calderon-Zygmund kernel, hence not integrable. In particular, the last integral must be understood in a weak sense: it can be defined rigorously through the decomposition

$$
\begin{aligned}
\int_{\mathbb{R}^{3}} & \int_{\mathbb{R}^{3}} \mathcal{M}(x-y) F(x, y) \rho(x) \rho(y) d x d y \\
:= & \int_{\mathbb{R}^{3}} \int_{\mathbb{R}^{3}} \mathcal{M}(x-y)[F(x, y)-F(y, y)] \rho(x) \rho(y) d x d y \\
& +\int_{\mathbb{R}^{3}}(\mathcal{M} \star \rho)(y) F(y, y) \rho(y) d y,
\end{aligned}
$$

where the first integral in the decomposition exists in the usual sense, while the second one is defined because $h \rightarrow \mathcal{M} \star h$ is continuous from $L^{p}\left(\mathbb{R}^{3}\right)$ to $L^{p}\left(\mathbb{R}^{3}\right)$ for any $1<p<\infty$ by the Calderon-Zygmund theorem. Of course, when $F$ is of the form $F(x, y)=f(x) g(y)$, one can write directly

$$
\int_{\mathbb{R}^{3}} \int_{\mathbb{R}^{3}} \mathcal{M}(x-y) F(x, y) \rho(x) \rho(y) d x d y=\int_{\mathbb{R}^{3}}(\mathcal{M} \star(\rho f)) \rho g,
$$

which allows us to give a meaning to $\left\langle\mu_{2, n}, f \otimes g\right\rangle$ for much less regular $f$ and $g$. Our theorem reads as follows.

Theorem 1.1. Let $\lambda>0, g \in L^{3+\varepsilon}, \varepsilon>0, \mu_{2} \in L^{\infty}\left(\mathbb{R}^{3}, \operatorname{Sym}\left(\operatorname{Sym}_{3, \sigma}(\mathbb{R})\right)\right)$. For all $n$, let $r_{n}$ be such that $\lambda=\frac{4 \pi}{3} n r_{n}^{3}, g_{n} \in L^{\frac{6}{5}}\left(\mathbb{R}^{3}\right)$. Let $u_{n, \lambda}$ be the solution of (1)-(2) in $\dot{H}^{1}\left(\mathbb{R}^{3}\right) \cap L^{6}\left(\mathbb{R}^{3}\right)$. Assume (A0)-(A1), that $g_{n} \rightarrow g$ in $L^{\frac{6}{5}}\left(\mathbb{R}^{3}\right)$, and that

$$
\mu_{2, n} \rightarrow \mu_{2}(x) \delta_{x=y} \quad \text { in } \mathscr{D}^{\prime}\left(\mathbb{R}^{3} \times \mathbb{R}^{3}, \operatorname{Sym}\left(\operatorname{Sym}_{3, \sigma}(\mathbb{R})\right)\right),
$$

with $\mu_{2, n}$ defined in (6). Then any accumulation point $u_{\lambda}$ of $u_{n, \lambda}$ solves

$$
\begin{cases}-\operatorname{div}\left(2\left[\mu+\frac{5}{2} \mu \rho \lambda+\mu_{2} \lambda^{2}\right] D\left(u_{\lambda}\right)-\mathbb{I} p_{\lambda}\right)=g+R_{\lambda} & \text { in } \mathbb{R}^{3}, \\ \operatorname{div}\left(u_{\lambda}\right)=0 & \text { in } \mathbb{R}^{3},\end{cases}
$$

where $R_{\lambda}$ satisfies, for all $q \geq 3$,

$$
\left|\left\langle R_{\lambda}, \phi\right\rangle\right| \leq C \lambda^{\frac{7}{3}}\|D \phi\|_{q} \quad \forall \phi \in \dot{H}^{1}\left(\mathbb{R}^{3}\right) \cap \dot{W}^{1, q}\left(\mathbb{R}^{3}\right) .
$$


A few remarks are in order.

Remark 1.1. One has directly from estimate (9) that for any $p \leq \frac{3}{2}$,

$$
\left\|u-u_{\lambda}\right\|_{\dot{W}^{1, p}\left(\mathbb{R}^{3}\right)} \leq C \lambda^{\frac{7}{3}},
$$

where $u$ is the solution of the effective model

$$
\begin{cases}-\operatorname{div}\left(2\left[\mu+\frac{5}{2} \mu \rho \lambda+\mu_{2} \lambda^{2}\right] D(u)-\mathbb{I} p\right)=g & \text { on } \mathbb{R}^{3} \\ \operatorname{div}(u)=0 & \text { on } \mathbb{R}^{3} .\end{cases}
$$

It follows by Sobolev embedding that $\left\|u-u_{\lambda}\right\|_{L_{\text {loc }}^{r}}=O\left(\lambda^{\frac{7}{3}}\right)$, for any $r=\frac{3 p}{3-p} \leq 3$. Moreover, as will be seen below, $\left(u_{n, \lambda}\right)_{n \in \mathbb{N}}$ is bounded in $\dot{H}^{1} \cap L^{6}$. Combining the last estimate with Rellich's theorem, it follows easily that

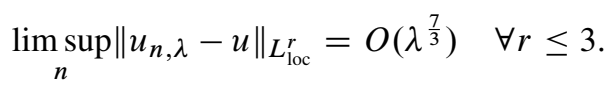

Remark 1.2. The main assumption of the theorem is the convergence of $\mu_{2, n}$ to $\mu_{2}(x) \delta_{x=y}$. With regard to the form of $\left\langle\mu_{2, n}, F\right\rangle$, cf. (7), this convergence corresponds to the local mean field limits alluded to above. In particular, the necessary condition (4) derived in [8] corresponds to the convergence of $\left\langle\mu_{2, n}, F\right\rangle$ for the special case $F(x, y)=1$.

The outline of the paper is the following. After preliminary results on the Stokes system, gathered in Section 2, we address the proof of Theorem 1.1 in Section 3. Finally, we turn in Section 4 to the discussion of assumption (A2). Roughly, we show that it is fulfilled by both periodic and random stationary particle distributions that satisfy the separation assumption (A1), and we discuss the corresponding limit $\mu_{2}$. There we closely follow the analysis in [8]. Notably, we show that when the distribution of particles is given by an isotropic process (plus technical conditions), then $\mu_{2} S: S=\frac{5}{2} \mu|S|^{2}$, a result that was not given in [8].

\section{Reminder on the Stokes problem}

In this section we recall some properties regarding the Stokes equation on an exterior domain. We denote by $(\mathcal{U}, \mathcal{P})$ the Green function of the Stokes equation:

$$
\mathcal{U}(x):=\frac{1}{8 \pi}\left(\frac{I_{3}}{|x|}+\frac{x \otimes x}{|x|^{3}}\right), \quad \mathcal{P}(x)=\frac{1}{4 \pi} \frac{x}{|x|^{3}} .
$$

Let $A \in \operatorname{Sym}_{3, \sigma}(\mathbb{R})$. We denote by $(V[A], Q[A])$ the solution to the Dirichlet problem

$$
\begin{cases}-\Delta u+\nabla p=0 & \text { on } \mathbb{R}^{3} \backslash B(0,1), \\ \operatorname{div}(u)=0 & \text { on } \mathbb{R}^{3} \backslash B(0,1), \\ u=-A x & \text { on } B(0,1),\end{cases}
$$


given by the explicit formulas

$$
\begin{aligned}
& V[A](x)=-\frac{5}{2} \frac{A: x \otimes x}{|x|^{5}} x-\frac{1}{|x|^{5}} A x+\frac{5}{2} \frac{(A: x \otimes x)}{|x|^{7}} x, \\
& Q[A](x)=-5 \frac{A: x \otimes x}{|x|^{5}} .
\end{aligned}
$$

An important feature of this solution is that, as easily seen from symmetry considerations, it fulfills the extra conditions

$$
\int_{\partial B(0,1)} \sigma(V[A], Q[A]) v=0, \quad \int_{\partial B(0,1)} x \times \sigma(V[A], Q[A]) v=0 .
$$

Moreover, we can link $V[A]$ to the Green function through the identity

$$
V[A](x)=\frac{20 \pi}{3} \nabla U(x) A+R[A](x),
$$

where $R[A]$ is homogeneous of degree -4 . Here, $\nabla \mathcal{U}$ is a third-rank tensor defined using the Einstein summation convention by

$$
\nabla \mathcal{U} A=\left(\partial x_{k} U_{i j} A_{j k}\right)_{1 \leq i \leq 3}=-\frac{3}{8 \pi} \frac{A: x \otimes x}{|x|^{5}} x .
$$

Moreover, a simple calculation yields the identity

$$
D(\nabla \mathcal{U} A): B=\frac{3}{8 \pi} \mathcal{M}(x) A: B=D(\nabla \mathcal{H}): A,
$$

where $\mathcal{M}$ was defined in (5), so that

$$
D(V[A])(x)=\frac{5}{2} \mathcal{M}(x) A+D(R[A])(x) .
$$

We also introduce the extensions

$$
\mathcal{V}[A](x)=\left\{\begin{array}{ll}
V[A] & \text { on } B(0,1)^{c}, \\
-A x & \text { on } B(0,1),
\end{array} \quad Q[A](x)= \begin{cases}Q[A] & \text { on } B(0,1)^{c}, \\
0 & \text { on } B(0,1) .\end{cases}\right.
$$

Direct computation shows that

$$
-\operatorname{div}(\sigma(\mathcal{V}[A], \mathcal{Q}[A]))=5 A x s^{1} \quad \text { in } \mathbb{R}^{3},
$$

where $s^{\eta}$ is the surface measure on the sphere of radius $\eta$.

We finish this part by recalling a classical estimate for the Stokes equation. Let $w \in$ $W^{1,2}\left(\bigcup B_{i}\right)$ be divergence-free. We consider the unique solution $(v, q)$ satisfying

$$
\begin{cases}-\operatorname{div}(2 D(v)-\mathbb{I} q)=0 & \text { on } \Omega_{n} \\ \operatorname{div}(v)=0 & \text { on } \Omega_{n} \\ D(v)=D(w) & \text { on } \bigcup B_{i}\end{cases}
$$


with the following conditions:

$$
\int_{\partial B_{i}} \sigma(v, q) n=0, \quad \int_{\partial B_{i}}[\sigma(v, q) n] \times\left(x-x_{i}\right)=0, \quad \forall 1 \leq i \leq n .
$$

Using integration by parts, one can show that $v$ is a minimizer of

$$
\left\{\int_{\mathbb{R}^{3}}|D(u)|^{2}, u \in \dot{H}_{\sigma}^{1}\left(\mathbb{R}^{3}\right) \text {, such that } D(u)=D(w) \text { on } \bigcup B_{i}\right\} .
$$

Combining this minimizing property with [15, Lem. 4.4] we have the following result:

Proposition 2.1. The unique solution $v$ of (22), (23) satisfies

$$
\|\nabla v\|_{L^{2}\left(\mathbb{R}^{3}\right)}^{2}=2\|D(v)\|_{L^{2}\left(\mathbb{R}^{3}\right)}^{2} \leq C\|D(w)\|_{L^{2}\left(\cup B_{i}\right)}^{2} .
$$

Proof. The first equality is well known: it follows from the identity $\Delta v=2 \operatorname{div}(D(v))$ and integration by parts. For the inequality, by the minimizing property of $v$, it is enough to construct a divergence-free velocity field $u$ that matches the condition $D(u)=D(w)$ on $\bigcup B_{i}$ and satisfies the same inequality. Classical considerations about the Bogovskii operator ensure the existence of fields $u_{i} \in H_{0}^{1}\left(\left(B\left(x_{i}, 2 r_{n}\right)\right)\right)$ such that

$$
\operatorname{div}\left(u_{i}\right)=0 \text { on } B\left(x_{i}, 2 r_{n}\right), \quad u_{i}=w-f_{B_{i}} w \text { on } B_{i},
$$

where, using the Poincaré-Wirtinger inequality, we get for all $i$,

$$
\left\|\nabla u_{i}\right\|_{H_{0}^{1}\left(B\left(x_{i}, 2 r_{n}\right)\right)} \leq C\|w-f w\|_{H^{1}\left(B_{i}\right)} \leq C^{\prime}\|\nabla w\|_{L^{2}\left(B_{i}\right)},
$$

with $C, C^{\prime}$ independent of $n$ by scaling considerations. See [11, Lem. 18] for details. We then take $u=\sum_{i} u_{i}$. Since the balls $B\left(x_{i}, 2 r_{n}\right)$ are disjoint by (A1) for $\lambda$ small enough, $u$ satisfies $D(u)=D(w)$ on $\bigcup B_{i}$ and we have

$$
\|u\|_{\dot{H}^{1}\left(\mathbb{R}^{3}\right)}^{2}=\sum_{i}\left\|\nabla u_{i}\right\|_{L^{2}\left(B\left(x_{i}, 2 r_{n}\right)\right)}^{2} \leq C \sum_{i}\|\nabla w\|_{L^{2}\left(B_{i}\right)}^{2} .
$$

Moreover, adding a proper rigid vector field to $w$ on each $B_{i}$, which does not change $D(w)$ on each $B_{i}$, we can always assume that $\int_{\partial B_{i}} w=\int_{\partial B_{i}} w \times\left(x-x_{i}\right)=0$. We conclude by applying [15, Lem. 4.4].

\section{Proof of Theorem 1.1}

By linearity of the Stokes equation, we can restrict to the case $\mu=1$. Let $q \geq 3$. The goal is to show that any accumulation point $u_{\lambda}$ of $u_{n, \lambda}$ satisfies a system of type (8) with remainder $R_{\lambda}$ satisfying

$$
\left\langle R_{\lambda}, \phi\right\rangle \leq C \lambda^{\frac{7}{3}}\|\phi\|_{W^{1, q}}
$$


for all divergence-free $\phi \in \dot{H}^{1} \cap W^{1, q}$. By density, it is enough to show such an inequality for all divergence-free $\phi \in C_{c}^{\infty}\left(\mathbb{R}^{3}\right)$. For any such $\phi$, we consider $\phi_{n}$ satisfying

$$
\begin{cases}-\operatorname{div}\left(2 D\left(\phi_{n}\right)-\mathbb{I} q_{n}\right)=2 \operatorname{div}\left(\left(\frac{5}{2} \rho \lambda+\lambda^{2} \mu_{2}\right) D(\phi)\right) & \text { on } \Omega_{n}, \\ \operatorname{div}\left(\phi_{n}\right)=0 & \text { on } \Omega_{n}, \\ \phi_{n}=\phi+\text { translation }+ \text { rotation } & \text { on } B_{i},\end{cases}
$$

with the following conditions:

$$
\begin{aligned}
\int_{\partial B_{i}} \sigma\left(\phi_{n}, q_{n}\right) v & =-2 \int_{\partial B_{i}}\left(\frac{5}{2} \rho \lambda+\mu_{2} \lambda^{2}\right) D(\phi) v d x \\
\int_{\partial B_{i}}\left[\sigma\left(u_{n}, p_{n}\right) v\right] \times\left(x-x_{i}\right) & =-2 \int_{\partial B_{i}}\left[\left(\frac{5}{2} \rho \lambda+\mu_{2} \lambda^{2}\right) D(\phi) v\right] \times\left(x-x_{i}\right) d x .
\end{aligned}
$$

Note that $\phi_{n}$ depends implicitly on $\lambda$. Similarly, we shall use $u_{n}$ instead of $u_{n, \lambda}$ for short. Testing $\phi-\phi_{n}$ in equation (1) we get

$$
\begin{aligned}
\int_{\Omega_{n}}\left(\phi-\phi_{n}\right) \cdot g_{n} & =-\int_{\Omega_{n}} \operatorname{div}\left(2 D\left(u_{n}\right)-\mathbb{I} p_{n}\right) \cdot\left(\phi-\phi_{n}\right) \\
& =2 \int_{\Omega_{n}} D\left(u_{n}\right): D\left(\phi-\phi_{n}\right)+\sum_{i} \int_{\partial B_{i}} \sigma\left(u_{n}, p_{n}\right) v \cdot\left(\phi-\phi_{n}\right) \\
& =2 \int_{\Omega_{n}} D\left(u_{n}\right): D(\phi)-2 \int_{\Omega_{n}} D\left(u_{n}\right): D\left(\phi_{n}\right)-\sum_{i} \int_{B_{i}} g_{n} \cdot\left(\phi-\phi_{n}\right) .
\end{aligned}
$$

We recall that in the second line of the above computations, the unit normal vector $v$ is pointing outward the balls. Using equations (24) and (25) we have

$$
\begin{aligned}
& -2 \int_{\Omega_{n}} D\left(u_{n}\right): D\left(\phi_{n}\right) \\
& \quad=\int_{\Omega_{n}} \operatorname{div}\left(2 D\left(\phi_{n}\right)-q_{n} \mathbb{I}\right) \cdot u_{n}+\sum_{i} \int_{\partial B_{i}}\left[\sigma\left(\phi_{n}, q_{n}\right) v\right] \cdot u_{n} \\
& \quad=-\int_{\Omega_{n}} 2 \operatorname{div}\left(\left(\frac{5}{2} \rho \lambda+\mu_{2} \lambda^{2}\right) D(\phi)\right) \cdot u_{n}+\sum_{i} \int_{\partial B_{i}}\left[\sigma\left(\phi_{n}, q_{n}\right) v\right] \cdot u_{n} \\
& \quad=2 \int_{\Omega_{n}}\left(\frac{5}{2} \rho \lambda+\mu_{2} \lambda^{2}\right) D(\phi): D\left(u_{n}\right) .
\end{aligned}
$$

We get the following relation for all $\phi$ using the fact that $D\left(u_{n}\right)=0$ on $B_{i}$ :

$$
2 \int_{\mathbb{R}^{3}} D\left(u_{n}\right):\left(1+\frac{5}{2} \rho \lambda+\mu_{2} \lambda^{2}\right) D(\phi)=\int_{\mathbb{R}^{3}} \phi \cdot g_{n}-\int_{\mathbb{R}^{3}} \phi_{n} \cdot g_{n} .
$$

By a simple energy estimate, $u_{n}$ also satisfies

$$
\int_{\mathbb{R}^{3}}\left|\nabla u_{n}\right|^{2}=2 \int_{\mathbb{R}^{3}}\left|D\left(u_{n}\right)\right|^{2}=\int_{\mathbb{R}^{3}} u_{n} \cdot g_{n} \leq\left\|u_{n}\right\|_{L^{6}}\left\|g_{n}\right\|_{L^{\frac{6}{5}}} \leq C\left\|\nabla u_{n}\right\|_{L^{2}},
$$


where the last inequality comes from the Sobolev embedding and the boundedness of $\left(g_{n}\right)_{n \in \mathbb{N}}$ in $L^{\frac{6}{5}}$. Hence, $\left(u_{n}\right)_{n \in \mathbb{N}}$ is bounded in $\dot{H}^{1} \cap L^{6}$. Denoting by $u_{\lambda}=\lim _{k} u_{n_{k}}$ an accumulation point, we deduce from (26) with $n=n_{k}$ that

$$
2 \int_{\mathbb{R}^{3}} D\left(u_{\lambda}\right):\left(1+\frac{5}{2} \rho \lambda+\mu_{2} \lambda^{2}\right) D(\phi)=\int_{\mathbb{R}^{3}} \phi \cdot g+\left\langle R_{\lambda}, \phi\right\rangle,
$$

where $\left\langle R_{\lambda}, \phi\right\rangle=-\lim _{k} \int_{\mathbb{R}^{3}} \phi_{n_{k}} \cdot g_{n_{k}}$. Note that this limit exists because all other terms in (26) converge when $n=n_{k}, k \rightarrow+\infty$. Moreover, it is clearly linear in $\phi$ as $\phi_{n}$ is linear in $\phi$. Furthermore, testing $\phi_{n}-\phi$ in (24), similar integrations by parts lead to

$2 \int_{\mathbb{R}^{3}}\left|D\left(\phi_{n}\right)\right|^{2}=2 \int_{\mathbb{R}^{3}} D\left(\phi_{n}\right): D(\phi)-2 \int_{\mathbb{R}^{3}}\left(\frac{5}{2} \lambda \rho+\mu_{2} \lambda^{2}\right) D(\phi):\left(D\left(\phi_{n}\right)-D(\phi)\right)$.

The Cauchy-Schwarz inequality implies that $\phi_{n}$ is bounded uniformly in $n$ in $\dot{H}^{1}$, hence in $L^{6}$. Eventually, as $g_{n} \rightarrow g$ strongly in $L^{\frac{6}{5}}$, we have $\left\langle R_{\lambda}, \phi\right\rangle=-\lim _{k} \int_{\mathbb{R}^{3}} \phi_{n_{k}} \cdot g$. Finally, to prove the theorem, it is enough to show that

$$
\forall q \geq 3, \quad \limsup _{n}\left|\int_{\mathbb{R}^{3}} \phi_{n} \cdot g\right| \leq C \lambda^{\frac{7}{3}}\|\phi\|_{W^{1, q}} .
$$

In order to obtain (27), we shall write $\left(\phi_{n}, q_{n}\right)=\left(\phi_{n}^{1}+\phi_{n}^{2}, q_{n}^{1}+q_{n}^{2}\right)$, where $\phi_{n}^{1}$ is a (somehow natural) approximation of $\phi_{n}$ and where $\phi_{n}^{2}$ is a remainder. Namely, we look for an approximation $\phi_{n}^{1}$ of the form

$$
\phi_{n}^{1}=\nabla \mathcal{U} \star\left(2\left(\lambda \frac{5}{2}|\mathcal{O}| \rho+\lambda^{2} \mu_{2}\right) D(\phi)\right)-r_{n} \sum_{i} \mathcal{V}\left[A_{i}\right]\left(\frac{x-x_{i}}{r_{n}}\right),
$$

where $\mathcal{U}, \mathcal{V}$ are defined in (11), (20). The rough idea behind this approximation is that the first term on the right-hand side should take care of the source term in (24), while the second term should take care of the boundary conditions at the balls $B_{i}$. In particular, the field $\phi_{\mathbb{R}^{3}}:=\nabla \mathcal{U} *\left(2\left(\lambda \frac{5}{2}|\mathcal{O}| \rho+\lambda^{2} \mu_{2}\right) D(\phi)\right)$ solves the Stokes equation

$$
-\Delta \phi_{\mathbb{R}^{3}}+\nabla q_{\mathbb{R}^{3}}=\operatorname{div}\left(2\left(\lambda \frac{5}{2}|\mathcal{O}| \rho+\lambda^{2} \mu_{2}\right) D(\phi)\right), \quad \operatorname{div} \phi_{\mathbb{R}^{3}}=0 \quad \text { in } \mathbb{R}^{3},
$$

while each term in the sum, that is, $\phi_{i, n}:=-r_{n} \mathcal{V}\left[A_{i}\right]\left(\frac{x-x_{i}}{r_{n}}\right)$, solves

$$
-\Delta \phi_{i, n}+\nabla q_{i, n}=0, \quad \operatorname{div} \phi_{i, n}=0 \text { in } \mathbb{R}^{3} \backslash B_{i},\left.\quad \phi_{i, n}\right|_{B_{i}}=A_{i}\left(x-x_{i}\right) .
$$

By looking at (24), it is tempting to take $A_{i}=D \phi_{i}$, where

$$
D \phi_{i}:=D(\phi)\left(x_{i}\right)
$$

as $\phi$ should be close to this value on the small ball $B_{i}$. However, this approximation is not accurate enough, and would only allow us to recover Einstein's formula. To go beyond, one must account for two extra contributions. The first one is the trace left at the balls 
by $\phi_{\mathbb{R}^{3}}$. More precisely, it will be enough to correct the trace of the $O(\lambda)$ term in $\phi_{\mathbb{R}^{3}}$. The second contribution is the trace left by all $\phi_{j, n}, j \neq i$, on ball $B_{i}$, which corresponds to binary interactions between particles. Again, it will be enough to account for the least decaying term in $\phi_{j, n}$; cf. decomposition (16). This leads to the following definition:

$$
\phi_{n}^{1}=\nabla \mathcal{U} *\left(2\left(\lambda \frac{5}{2} \rho+\lambda^{2} \mu_{2}\right) D(\phi)\right)-r_{n} \sum_{i} \mathcal{V}\left[D \phi_{i}+S_{i}^{1}+S_{i}^{2}\right]\left(\frac{x-x_{i}}{r_{n}}\right),
$$

where, using relation (18),

$$
\begin{aligned}
S_{i}^{1} & :=-f_{B_{i}} D(\nabla U *(\lambda 5|\mathcal{O}| \rho D(\phi)))(x) d x=-\frac{15}{8 \pi} \lambda f_{B_{i}}(\mathcal{M} *(\rho D(\phi)))(x) d x \\
& =-\frac{15}{8 \pi} \lambda f_{B_{i}}\left(\int_{\mathbb{R}^{3}} \mathcal{M}(x-y) D(\phi)(y) \rho(y) d y\right) d x,
\end{aligned}
$$

while, still using (18),

$$
\begin{aligned}
S_{i}^{2} & :=\sum_{j \neq i} f_{B_{i}} \frac{20 \pi}{3} D\left(\nabla U D \phi_{j}\right)\left(\frac{x-x_{j}}{r_{n}}\right) d x=\frac{5}{2} r_{n}^{3} f_{B_{i}} \sum_{j \neq i} \mathcal{M}\left(x-x_{j}\right) D \phi_{j} d x \\
& =\frac{15}{8 \pi} \frac{\lambda}{n} \sum_{j \neq i} f_{B_{i}} \mathcal{M}\left(x-x_{j}\right) D \phi_{j} d x .
\end{aligned}
$$

Direct computations using formula (21) yield

$$
\begin{aligned}
& -\operatorname{div}\left(\sigma\left(\phi_{n}^{1}, q_{n}^{1}\right)\right) \\
& =\operatorname{div}\left(2\left(\frac{5}{2} \rho \lambda+\mu_{2} \lambda^{2}\right) D(\phi)\right)-5 \sum_{i}\left(D \phi_{i}+S_{i}^{1}+S_{i}^{2}\right) \nabla 1_{B_{i}} \\
& \quad=\operatorname{div}\left(\left(5 \rho \lambda+2 \mu_{2} \lambda^{2}\right) D(\phi)-\frac{5 \lambda}{n} \sum_{i} \frac{1}{\left|B_{i}\right|} 1_{B_{i}}\left(D \phi_{i}+S_{i}^{1}+S_{i}^{2}\right)\right) \quad \text { in } \mathbb{R}^{3}
\end{aligned}
$$

Using the definitions of $S_{i}^{1}$ and $S_{i}^{2},(29)$ and (30), we find

$$
\begin{aligned}
-\operatorname{div}\left(\sigma\left(\phi_{n}^{1}, q_{n}^{1}\right)\right) & \\
=\operatorname{div}( & \left(5 \lambda \rho+2 \lambda^{2} \mu_{2}\right) D(\phi)-\frac{5 \lambda}{n} \sum_{i} \frac{1}{\left|B_{i}\right|} 1_{B_{i}} D \phi_{i} \\
& -\frac{5 \lambda}{n} \sum_{i} \frac{1}{\left|B_{i}\right|} 1_{B_{i}}\left(-\frac{15 \pi}{8} \lambda f_{B_{i}} \int_{\mathbb{R}^{3}} \mathcal{M}(x-y) D(\phi)(y) \rho(y) d y d x\right) \\
& \left.-\frac{5 \lambda}{n} \sum_{i} \frac{1}{\left|B_{i}\right|} 1_{B_{i}}\left(-\frac{15 \pi}{8} \frac{\lambda}{n} f_{B_{i}} \sum_{j \neq i} \mathcal{M}\left(x-x_{j}\right) D \phi_{j} d x\right)\right)
\end{aligned}
$$




$$
\begin{aligned}
& =5 \lambda \operatorname{div}\left(\rho D(\phi)-\frac{1}{n} \sum_{i} \frac{1}{\left|B_{i}\right|} 1_{B_{i}} D \phi_{i}\right) \\
& +2 \lambda^{2} \operatorname{div}\left(\mu_{2} D(\phi)-\frac{75}{16 \pi} \frac{1}{n^{2}} \sum_{i} \frac{1}{\left|B_{i}\right|} 1_{B_{i}} \sum_{j \neq i} f_{B_{i}} \mathcal{M}\left(x-x_{j}\right) D \phi_{j} d x\right. \\
& \left.\quad+\frac{75}{16 \pi} \frac{1}{n} \sum_{i} \frac{1}{\left|B_{i}\right|} 1_{B_{i}} f_{B_{i}} \int_{\mathbb{R}^{3}} \mathcal{M}(x-y) D(\phi)(y) \rho(y) d y d x\right) \text { in } \mathbb{R}^{3} .
\end{aligned}
$$

Moreover, thanks to property (15), it is easily seen that

$$
\begin{aligned}
\int_{\partial B_{i}} \sigma\left(\phi_{n}^{1}, q_{n}^{1}\right) \nu & =-2 \int_{\partial B_{i}}\left(\frac{5}{2} \rho \lambda+\mu_{2} \lambda^{2}\right) D(\phi) v d x \\
\int_{\partial B_{i}} x \times\left(\sigma\left(\phi_{n}^{1}, q_{n}^{1}\right) \nu\right) & =-2 \int_{\partial B_{i}}\left(\left(\frac{5}{2} \rho \lambda+\mu_{2} \lambda^{2}\right) D(\phi) v\right) \times\left(x-x_{i}\right) d x .
\end{aligned}
$$

It follows that $\left(\phi_{n}^{2}, q_{n}^{2}\right)$ satisfies

$$
\begin{gathered}
\operatorname{div}\left(\sigma\left(\phi_{n}^{2}, q_{n}^{2}\right)\right)=0 \text { on } \Omega_{n}, \quad \int_{\partial B_{i}} \sigma\left(\phi_{n}^{2}, p_{n}^{2}\right) v=0, \\
\int_{\partial B_{i}} \sigma\left(\phi_{n}^{2}, p_{n}^{2}\right) v \times\left(x-x_{i}\right)=0
\end{gathered}
$$

with boundary condition

$$
D\left(\phi_{n}^{2}\right)=D\left(\psi_{n}^{2}+\widetilde{\psi}_{n}^{2}\right) \quad \text { on } \bigcup B_{i},
$$

where $\psi_{n}^{2}$ and $\widetilde{\psi}_{n}^{2}$ are defined by

$$
\left.\psi_{n}^{2}(x)\right|_{B_{i}}:=\phi(x)-D \phi_{i} \cdot\left(x-x_{i}\right), \quad x \in B_{i}
$$

and

$$
\begin{aligned}
\widetilde{\psi}_{n}^{2}(x)=-\left(S_{i}^{1}+S_{i}^{2}\right) \cdot\left(x-x_{i}\right)- & \left(\nabla U *\left(2\left(\frac{5}{2} \rho \lambda+\mu_{2} \lambda^{2}\right) D(\phi)\right)\right. \\
& \left.-r_{n} \sum_{j \neq i} V\left[D \phi_{j}+S_{j}^{1}+S_{j}^{2}\right]\left(\frac{x-x_{j}}{r_{n}}\right)\right), \quad x \in B_{i} .
\end{aligned}
$$

We aim now to estimate both terms $\int g \phi_{n}^{1}$ and $\int g \phi_{n}^{2}$.

\subsection{Estimate of $\phi_{n}^{2}$}

Most of this subsection is dedicated to the derivation of the following proposition:

Proposition 3.1. For all $q \geq 2$,

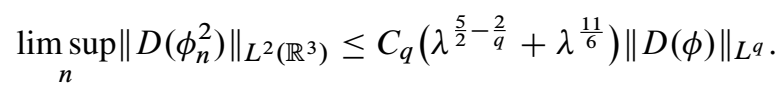


Before we prove this proposition, we show that it implies the following result:

Proposition 3.2. For all $q \geq 2$,

$$
\limsup _{n}\left|\int_{\mathbb{R}^{3}} g \phi_{n}^{2}\right| \leq C_{g, q} \lambda^{\frac{1}{2}}\left(\lambda^{\frac{5}{2}-\frac{2}{q}}+\lambda^{\frac{11}{6}}\right)\|D(\phi)\|_{L^{q}} .
$$

Note the extra factor $\lambda^{1 / 2}$ compared to Proposition 3.1, which is crucial to obtain an $o\left(\lambda^{2}\right)$ error. Note also that for $q \geq 3$, this term is bounded by $\lambda^{\frac{7}{3}}\|D(\phi)\|_{L^{q}}$.

Proof of Proposition 3.2. We introduce the solution $u_{g}$ of the Stokes equation

$$
-\Delta u_{g}+\nabla p_{g}=g, \quad \operatorname{div} g=0, \quad \text { in } \mathbb{R}^{3} .
$$

As $g \in L^{3+\varepsilon}, u_{g} \in W_{\text {loc }}^{2,3+\varepsilon}$, so that $D\left(u_{g}\right)$ is continuous. Integrations by parts yield

$$
\begin{aligned}
\int_{\mathbb{R}^{3}} g \phi_{n}^{2} & =\int_{\mathbb{R}^{3}}\left(-\Delta u_{g}+\nabla p_{g}\right) \phi_{n}^{2}=2 \int_{\mathbb{R}^{3}} D\left(u_{g}\right): D\left(\phi_{n}^{2}\right) \\
& =2 \int_{\cup B_{i}} D\left(u_{g}\right): D\left(\phi_{n}^{2}\right)-\sum_{i} \int_{\partial B_{i}} u_{g} \cdot \sigma\left(\phi_{n}^{2}, q_{n}^{2}\right) v \\
& =2 \int_{\cup B_{i}} D\left(u_{g}\right): D\left(\phi_{n}^{2}\right)-\sum_{i} \int_{\partial B_{i}}\left(u_{g}+u_{g}^{i}+\omega_{g}^{i} \times\left(x-x_{i}\right)\right) \cdot \sigma\left(\phi_{n}^{2}, q_{n}^{2}\right) v
\end{aligned}
$$

for any constant vectors $u_{g}^{i}, \omega_{g}^{i}, 1 \leq i \leq n$, by the last two relations in (32). As $u_{g}+u_{g}^{i}+\omega_{g}^{i} \times\left(x-x_{i}\right)$ is divergence-free, one has

$$
\int_{\partial B_{i}}\left(u_{g}+u_{g}^{i}+\omega_{g}^{i} \times\left(x-x_{i}\right)\right) \cdot v=0 .
$$

We can apply classical considerations on the Bogovskii operator ([6]): for any $1 \leq i \leq n$, there exists $U_{g}^{i} \in H_{0}^{1}\left(B\left(x_{i}, 2 r_{n}\right)\right)$ such that

$$
\operatorname{div} U_{g}^{i}=0 \text { in } B\left(x_{i}, 2 r_{n}\right), \quad U_{g}^{i}=u_{g}+u_{g}^{i}+\omega_{g}^{i} \times\left(x-x_{i}\right) \text { in } B_{i},
$$

and with

$$
\left\|\nabla U_{g}^{i}\right\|_{L^{2}} \leq C_{i, n}\left\|u_{g}+u_{g}^{i}+\omega_{g}^{i} \times\left(x-x_{i}\right)\right\|_{W^{1,2}\left(B_{i}\right)} .
$$

Furthermore, by a proper choice of $u_{g}^{i}$ and $\omega_{g}^{i}$, we can ensure the Korn inequality:

$$
\left\|u_{g}+u_{g}^{i}+\omega_{g}^{i} \times\left(x-x_{i}\right)\right\|_{W^{1,2}\left(B_{i}\right)} \leq c_{i, n}^{\prime}\left\|D\left(u_{g}\right)\right\|_{L^{2}\left(B_{i}\right)},
$$

resulting in

$$
\left\|\nabla U_{g}^{i}\right\|_{L^{2}} \leq C\left\|D\left(u_{g}\right)\right\|_{L^{2}\left(B_{i}\right)},
$$

where the constant $C$ in the last inequality can be taken independent of $i$ and $n$ by translation and scaling arguments. Extending $U_{g}^{i}$ by zero, and denoting $U_{g}=\sum U_{g}^{i}$, we have for $d_{n}>4 r_{n}$ (which is implied by (A1) for $\lambda$ small enough):

$$
\left\|\nabla U_{g}\right\|_{L^{2}} \leq C\left\|D\left(u_{g}\right)\right\|_{L^{2}\left(\cup B_{i}\right)} .
$$


Back to our calculation, we find

$$
\begin{aligned}
\int_{\mathbb{R}^{3}} g \phi_{n}^{2} & =2 \int_{\cup B_{i}} D\left(U_{g}\right): D\left(\phi_{n}^{2}\right)-\sum_{i} \int_{\partial B_{i}} U_{g} \cdot \sigma\left(\phi_{n}^{2}, q_{n}^{2}\right) v \\
& =2 \int_{\mathbb{R}^{3}} D\left(U_{g}\right): D\left(\phi_{n}^{2}\right) .
\end{aligned}
$$

By using (37) and the Cauchy-Schwarz inequality, we obtain

$$
\left|\int_{\mathbb{R}^{3}} g \phi_{n}^{2}\right| \leq C\left\|D\left(u_{g}\right)\right\|_{L^{2}\left(\cup B_{i}\right)}\left\|D\left(\phi_{n}^{2}\right)\right\|_{L^{2}\left(\mathbb{R}^{3}\right)} \leq C\left\|D\left(u_{g}\right)\right\|_{L^{\infty}} \lambda^{\frac{1}{2}}\left\|D\left(\phi_{n}^{2}\right)\right\|_{L^{2}\left(\mathbb{R}^{3}\right)},
$$

so that combining with Proposition 3.1 yields the result.

We now turn to the proof of Proposition 3.1. According to the definition of $\phi_{n}^{2}$ in (32), (33), Proposition 2.1 implies

$$
\left\|\nabla \phi_{n}^{2}\right\|_{L^{2}\left(\Omega_{n}\right)}^{2} \leq C\left(\left\|D\left(\psi_{n}^{2}\right)\right\|_{L^{2}\left(\cup B_{i}\right)}^{2}+\left\|D\left(\widetilde{\psi}_{n}^{2}\right)\right\|_{L^{2}\left(\cup B_{i}\right)}^{2}\right) .
$$

As regards $\psi_{n}^{2}$, defined in (34), we compute

$$
\begin{aligned}
\left\|D\left(\psi_{n}^{2}\right)\right\|_{L^{2}\left(\cup B_{i}\right)}^{2} & =\sum_{i} \int_{B_{i}}\left|D(\phi)-D \phi_{i}\right|^{2} d x \\
& \leq\left\|\nabla^{2} \phi\right\|_{\infty}^{2} \sum_{i} \int_{B_{i}} r_{n}^{2} d x \leq\left\|\nabla^{2} \phi\right\|_{\infty}^{2} \lambda r_{n}^{2} \underset{n \rightarrow \infty}{\longrightarrow} 0 .
\end{aligned}
$$

As regards $\widetilde{\psi}_{n}^{2}$, defined in (35), we use identities (18) and (19) to write for all $x \in B_{i}$,

$$
\begin{aligned}
D\left(\widetilde{\psi}_{n}^{2}\right)(x)= & -\frac{15}{8 \pi} \lambda \mathcal{M} *(\rho D(\phi))(x)-S_{i}^{1} \\
& +\frac{15 \lambda}{8 \pi n} \sum_{j \neq i} \mathcal{M}\left(x-x_{j}\right) D \phi_{j}-S_{i}^{2} \\
& +r_{n}^{2} \frac{3 \lambda}{4 \pi n} \sum_{j \neq i} D\left(R\left[D \phi_{j}\right]\right)\left(x-x_{j}\right) \\
& -\frac{3 \pi}{4} \lambda^{2} \mathcal{M} *\left(\mu_{2} D(\phi)\right)(x) \\
& +\sum_{j \neq i} D\left(V\left[S_{j}^{1}+S_{j}^{2}\right]\right)\left(\frac{x-x_{j}}{r_{n}}\right)=\sum_{i=1}^{5} E_{i}(x) .
\end{aligned}
$$

For $E_{4}$ we have for all $q \in[2, \infty)$,

$$
\begin{aligned}
\left\|E_{4}\right\|_{L^{2}\left(\cup B_{i}\right)}^{2} & =\frac{9 \pi^{2}}{16} \lambda^{4}\left\|\mathcal{M} *\left(\mu_{2} D(\phi)\right)\right\|_{L^{2}\left(\bigcup B_{i}\right)}^{2} \\
& \leq \frac{9 \pi^{2}}{16} \lambda^{4}\left\|\mathcal{M} *\left(\mu_{2} D(\phi)\right)\right\|_{L^{q}}^{2}\left|\bigcup B_{i}\right|^{1-\frac{2}{q}} \\
& \leq C \lambda^{4}\left\|\mu_{2}\right\|_{\infty}^{2} \lambda^{1-\frac{2}{q}}\|D(\phi)\|_{L^{q}}^{2},
\end{aligned}
$$


because $\mathcal{M}$ is a Calderon-Zygmund operator. The estimate of $E_{5}$ is more difficult. We shall rely on (A1), and notably make crucial use of the following lemma, taken from [8]:

Lemma 3.3 ([8, Lem. 2.4 $\left.]^{1}\right)$. Under assumption (A1), for all $q \in(1, \infty)$, there exists $C>0$, such that for all $A_{1}, \ldots, A_{n}$ in $\operatorname{Sym}_{3, \sigma}(\mathbb{R})$,

$$
\sum_{i}\left|\sum_{j \neq i} r_{n}^{3} \mathcal{M}\left(x_{i}-x_{j}\right) A_{j}\right|^{q} \leq C \lambda^{q-1} \sum_{i}\left|A_{i}\right|^{q},
$$

as well as

$$
\sum_{i}\left|\sum_{j \neq i} r_{n}^{3} f \mathcal{M}\left(x-x_{j}\right) d x A_{j}\right|^{q} \leq C \lambda^{q-1} \sum_{i}\left|A_{i}\right|^{q} .
$$

In particular, this lemma can be applied to matrices $A_{j}=S_{j}^{2}$; cf. (30). We find

$$
\begin{aligned}
\sum_{i}\left|S_{i}^{2}\right|^{q} & \leq C \sum_{i}\left|\sum_{j \neq i} r_{n}^{3} f_{B_{i}} \mathcal{M}\left(x-x_{j}\right) d x D \phi_{j}\right|^{q} \\
& \leq C \lambda^{q-1} \sum_{i}\left|D \phi_{j}\right|^{q},
\end{aligned}
$$

where in this computation and all computations below, the constant $C$ may change from line to line. Moreover, by (A0),

$$
\frac{1}{n} \sum_{i}\left|D \phi_{j}\right|^{q} \underset{n \rightarrow+\infty}{\longrightarrow} \int_{\mathbb{R}^{3}}|D(\phi)|^{q}(x) \rho(x) d x
$$

so that

$$
\underset{n}{\limsup } \frac{1}{n} \sum_{i}\left|S_{i}^{2}\right|^{q} \leq C \lambda^{q-1}\|D(\phi)\|_{L^{q}}^{q} .
$$

Besides Lemma 3.3, we shall also make use of the following easy generalization of Young's inequality:

$$
\forall q \geq 1, \quad \sum_{i}\left(\sum_{j}\left|a_{i j} b_{j}\right|\right)^{q} \leq \max \left(\sup _{i} \sum_{j}\left|a_{i j}\right|, \sup _{j} \sum_{i}\left|a_{i j}\right|\right)^{q} \sum_{i}\left|b_{i}\right|^{q} .
$$

We now introduce $y_{i}:=x_{i} n^{-1 / 3}$ so that $\left|y_{i}-y_{j}\right| \geq \frac{1}{2}\left(c+\left|y_{i}-y_{j}\right|\right)$ with $c$ the constant appearing in (A1). Using decomposition (19) and the homogeneity of each term in this decomposition, we obtain, for all $q \geq 2$,

$$
\begin{aligned}
\left\|E_{5}\right\|_{L^{2}\left(\cup B_{i}\right)}^{2} & \leq \lambda^{1-\frac{2}{q}}\left\|E_{5}\right\|_{L^{q}\left(\cup B_{i}\right)}^{2} \\
& \leq C \lambda^{1-\frac{2}{q}}\left(\sum_{i} \int_{B_{i}}\left|\sum_{j \neq i} D\left(V\left[S_{j}^{1}+S_{j}^{2}\right]\right)\left(\frac{x-x_{j}}{r_{n}}\right)\right|^{q} d x\right)^{\frac{2}{q}}
\end{aligned}
$$

\footnotetext{
${ }^{1}$ Only the first inequality is stated in [8, Lem. 2.4], but a look at the proof shows that it follows from the second one.
} 


$$
\begin{aligned}
\leq & C \lambda^{1-\frac{2}{q}}\left(\sum_{i}\left|B_{1}\right|\left|\sum_{j \neq i} r_{n}^{3} \mathcal{M}\left(S_{j}^{1}+S_{j}^{2}\right)\left(x_{i}-x_{j}\right)\right|^{q} d x\right)^{\frac{2}{q}} \\
& +C \lambda^{1-\frac{2}{q}} r_{n}^{8}\left(\sum_{i}\left|B_{1}\right|\left|\sum_{j \neq i} \frac{\left|S_{j}^{1}+S_{j}^{2}\right|}{\left|x_{i}-x_{j}\right|^{4}}\right|^{q}\right)^{\frac{2}{q}} \\
\leq & C \lambda^{1-\frac{2}{q}}\left(\sum_{i}\left|B_{1}\right|\left|\sum_{j \neq i} r_{n}^{3} \mathcal{M}\left(S_{j}^{1}+S_{j}^{2}\right)\left(x_{i}-x_{j}\right)\right|^{q} d x\right)^{\frac{2}{q}} \\
& +C \lambda^{1-\frac{2}{q}} \lambda^{\frac{8}{3}}\left(\sum_{i}\left|B_{1}\right|\left|\sum_{j} \frac{\left|S_{j}^{1}+S_{j}^{2}\right|}{c+\left|y_{i}-y_{j}\right|^{4}}\right|^{q}\right)^{\frac{2}{q}} .
\end{aligned}
$$

We can then apply Lemma 3.3 to the first term, and apply (43) to the second term (together with the fact that $\sum_{i} \frac{1}{c+\left|y_{i}-y_{j}\right|^{4}}$ is uniformly bounded in $j$ ). We obtain

$$
\begin{array}{rl}
\left\|E_{5}\right\|_{L^{2}\left(\cup B_{i}\right) \leq}^{2} & C \lambda^{1-\frac{2}{q}}\left(\left|B_{1}\right| \lambda^{q-1} \sum_{i}\left|S_{i}^{1}+S_{i}^{2}\right|^{q}\right)^{\frac{2}{q}} \\
& +C \lambda^{\frac{8}{3}} \lambda^{1-\frac{2}{q}}\left(\sum_{i}\left|B_{1}\right|\left|S_{i}^{1}+S_{i}^{2}\right|^{q}\right)^{\frac{2}{q}} \\
\leq & C \lambda^{3-\frac{2}{q}}\left(\frac{1}{n} \sum_{i}\left|S_{i}^{1}+S_{i}^{2}\right|^{q}\right)^{2 / q}
\end{array}
$$

for any $q \geq 2$, where the last bound comes from Hölder's inequality. It remains to bound $\frac{1}{n} \sum_{i}\left|S_{i}^{1}+S_{i}^{2}\right|^{q}$. By (29), we have

$$
\left|S_{i}^{1}\right|^{q} \leq C \frac{\lambda^{q}}{\left|B_{1}\right|^{q}}\left(\int_{B_{i}}|M \star(\rho D(\phi))|\right)^{q} \leq C \frac{\lambda^{q}}{\left|B_{1}\right|} \int_{B_{i}}|M \star(\rho D(\phi))|^{q},
$$

so that

$$
\frac{1}{n} \sum_{i}\left|S_{i}^{1}\right|^{q} \leq C \lambda^{q-1}\|M \star(\rho D(\phi))\|_{L^{q}}^{q} \leq C \lambda^{q-1}\|D(\phi)\|_{L^{q}}^{q},
$$

where we used the $L^{q}$ continuity of the convolution with $\mathcal{M}$ again. Combining this inequality with (42), and injecting in the bound for $E_{5}$, we get that for all $q \geq 2$,

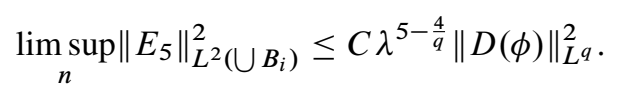

We now turn to $E_{3}$. We use the fact that $D(R[A])(x)=O\left(|A||x|^{-5}\right)$. We find

$$
\begin{aligned}
\left\|E_{3}\right\|_{L^{2}\left(\cup B_{i}\right)}^{2} & \leq C\left|B_{1}\right| \sum_{i}\left|\lambda^{\frac{5}{3}} \sum_{j \neq i} \frac{\left|D \phi_{j}\right|}{\left(c+\left|y_{i}-y_{j}\right|\right)^{5}}\right|^{2} \\
& \leq C\left|B_{1}\right| \lambda^{\frac{10}{3}} \sum_{i}\left|D \phi_{j}\right|^{2}
\end{aligned}
$$


where the last line follows from (43). Using again (41), we get eventually

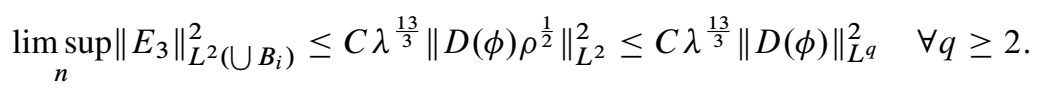

We recall now the expressions for $E_{1}$ and $E_{2}$ on $B_{i}$ :

$$
\begin{aligned}
& E_{1}(x)=-\frac{15}{8 \pi} \lambda\left(\int_{\mathbb{R}^{3}} \mathcal{M}(x-y) D(\phi)(y) \rho(y) d y\right. \\
&\left.-f_{B_{i}} \int_{\mathbb{R}^{3}} \mathcal{M}(z-y) D(\phi)(y) \rho(y) d y d z\right), \\
& E_{2}(x)=\frac{15}{8 \pi} \lambda\left(\frac{1}{n} \sum_{j \neq i} \mathcal{M}\left(x-x_{j}\right) D \phi_{j}-f_{B_{i}} \frac{1}{n} \sum_{j \neq i} \mathcal{M}\left(z-x_{j}\right) D \phi_{j} d z\right) .
\end{aligned}
$$

We claim first that

$$
\limsup _{n}\left\|E_{1}\right\|_{L^{2}\left(\cup B_{i}\right)}=0 .
$$

To prove (46), the idea is to regularize $D(\phi) \rho$ using a convolution with a mollifier $\chi_{\eta}$. Denoting by \|\|$_{0, \mu}$ the Hölder seminorm of exponent $\mu$, we get

$$
\begin{aligned}
\left\|(D(\phi) \rho) \star \chi_{\eta}\right\|_{0, \mu} & \leq C\left\|(D(\phi) \rho) \star \chi_{\eta}\right\|_{L^{\infty}}^{1-\mu}\left\|(D(\phi) \rho) \star \nabla \chi_{\eta}\right\|_{L^{\infty}}^{\mu} \\
& \leq \frac{C}{\eta^{\mu+\frac{3}{q}}}\|D(\phi) \rho\|_{L^{q}}
\end{aligned}
$$

for all $q \geq 1$. Hence we get, using the fact that $\mathcal{M}$ is a Zygmund-Calderon kernel, for all $q \geq 2$ and any $\mu \in(0,1)$,

$$
\begin{aligned}
\sum_{i} \int_{B_{i}}\left|E_{1}(x)\right|^{2} d x \leq & C \lambda^{2} \sum_{i} \int_{B_{i}}\left|\mathcal{M} \star\left(D(\phi) \rho-D(\phi) \rho \star \chi_{\eta}\right)\right|^{2} \\
& +C \lambda^{2} n\left|B_{1}\right| r_{n}^{2 \mu}\left\|(D(\phi) \rho) * \chi_{\eta}\right\|_{0, \mu}^{2} \\
\leq & \lambda^{3-\frac{2}{q}}\left\|D(\phi) \rho-(D(\phi) \rho) * \chi_{\eta}\right\|_{q}^{2}+\lambda^{3} \frac{r_{n}^{2 \mu}}{\eta^{2 \mu+6 / q}}\|D(\phi) \rho\|_{L^{q}}^{2},
\end{aligned}
$$

which vanishes when taking the lim sup in $n$ for fixed $\eta$ and then taking the limit $\eta \rightarrow 0$.

Finally, we bound $E_{2}$ by

$$
\begin{aligned}
\left\|E_{2}\right\|_{L^{2}\left(\cup B_{i}\right)}^{2} & \leq C \frac{\lambda^{2}}{n^{2}} \sum_{i} \int_{B_{i}}\left|\sum_{j \neq i}\left(\sup _{z \in B_{i}}\left|\nabla \mathcal{M}\left(z-x_{j}\right)\right|\right) r_{n}\right| D \phi_{j}||^{2} \\
& \leq C \frac{\lambda^{3}}{n^{3}} \sum_{i}\left|\sum_{j \neq i} \frac{r_{n}}{\left|x_{i}-x_{j}\right|^{4}}\right| D \phi_{j}||^{2} \\
& \leq C \lambda^{\frac{11}{3}} \frac{1}{n} \sum_{i}\left|\sum_{j} \frac{1}{\left(c+\left|y_{i}-y_{j}\right|\right)^{4}}\right| D \phi_{j}||^{2} \\
& \leq C \lambda^{\frac{11}{3}} \frac{1}{n} \sum_{i}\left|D \phi_{i}\right|^{2},
\end{aligned}
$$


where the last line comes from (43). We obtain

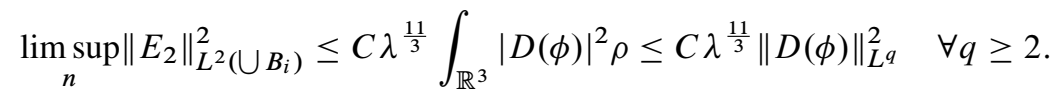

By the combination of (46), (47), (45), (40), (44), we find

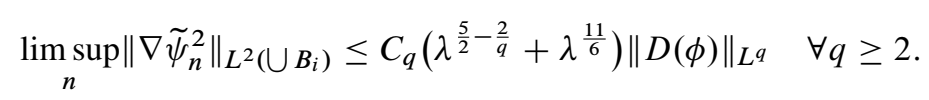

This inequality, together with (39) and (38), concludes the proof of Proposition 3.1.

\subsection{Estimate of $\phi_{n}^{1}$}

The goal of this subsection is to show the following proposition:

Proposition 3.4. For all $q \geq 1$,

$$
\limsup _{n}\left|\int_{\mathbb{R}^{3}} g \phi_{n}^{1}\right| \leq C \lambda^{\frac{7}{3}}\|D(\phi)\|_{L^{q}} .
$$

The main estimate (27) is then an easy consequence of Propositions 3.2 and 3.4, which, as explained before (27), concludes the proof of Theorem 1.1.

The derivation of Proposition 3.4 will rely strongly on our assumption (A2). First, let us recall that $\phi_{n}$ is bounded uniformly in $n$ in $\dot{H}^{1}$, and so is $\phi_{n}^{2}$ by Proposition 3.1. It follows that $\phi_{n}^{1}$ is bounded uniformly in $n$ in $\dot{H}^{1}$. Hence, by a density argument, it is enough to establish the inequality in Proposition 3.4 for a $C_{c}^{\infty}$ field $g$, as long as the constant $C$ on the right-hand side only involves $\|g\|_{L^{3+\varepsilon}}$. From now on, we assume $g$ to be smooth and compactly supported.

We introduce again the solution $\left(u_{g}, p_{g}\right)$ of (36). As $g$ is smooth, so is $u_{g}$. Moreover, by elliptic regularity and Sobolev embedding, we have for a compact $K$ containing all the balls,

$$
\left\|D\left(u_{g}\right)\right\|_{L^{\infty}(K)} \lesssim\left\|D\left(u_{g}\right)\right\|_{W^{1,3+\varepsilon}(K)} \lesssim\|g\|_{L^{3+\varepsilon}} .
$$

With (31), we compute

$$
\begin{aligned}
\int_{\mathbb{R}^{3}} \phi_{n}^{1} \cdot g= & \int_{\mathbb{R}^{3}} \phi_{n}^{1} \cdot\left(-\Delta u_{g}+\nabla p_{g}\right)=\int_{\mathbb{R}^{3}} D\left(\phi_{n}^{1}\right): D\left(u_{g}\right) \\
= & -\int_{\mathbb{R}^{3}} \operatorname{div}\left(\sigma\left(\phi_{n}^{1}, q_{n}^{1}\right)\right) \cdot u_{g} \\
= & -5 \lambda \int_{\mathbb{R}^{3}}\left(\rho D(\phi)-\frac{1}{n} \sum_{i} \frac{1}{\left|B_{i}\right|} 1_{B_{i}} D \phi_{i}\right): D\left(u_{g}\right) \\
& -2 \lambda^{2} \int_{\mathbb{R}^{3}}\left(\mu_{2} D(\phi)-\frac{75}{16 \pi} \frac{1}{n^{2}} \sum_{i} \frac{1}{\left|B_{i}\right|} 1_{B_{i}} \sum_{j \neq i} f_{B_{i}} \mathcal{M}\left(x-x_{j}\right) D \phi_{j} d x\right. \\
& \left.+\frac{75}{16 \pi} \frac{1}{n} \sum_{i} \frac{1}{\left|B_{i}\right|} 1_{B_{i}} f_{B_{i}} \int_{\mathbb{R}^{3}} \mathcal{M}(x-y) D(\phi)(y) \rho(y) d y d x\right): D\left(u_{g}\right) .
\end{aligned}
$$


We set

$$
\begin{aligned}
\bar{\mu}_{2, n}:=\frac{75}{16 \pi}( & \frac{1}{n^{2}} \sum_{i \neq j}\left(f_{B_{i}} \mathcal{M}\left(\cdot-x_{j}\right)\right) \frac{1}{\left|B_{i}\right|} 1_{B_{i}}(x) d x \delta_{y_{j}}(d y) \\
& \left.-\sum_{i} \frac{1}{n}\left(f_{B_{i}} \mathcal{M}(\cdot-y)\right) \frac{1}{\left|B_{i}\right|} 1_{B_{i}}(x) d x \rho(y) d y\right) .
\end{aligned}
$$

See (6) for comparison. We have

$$
\begin{aligned}
\int_{\mathbb{R}^{3}} \phi_{n}^{1} \cdot g= & -5 \lambda \int_{\mathbb{R}^{3}}\left(\rho D(\phi)-\frac{1}{n} \sum_{i} \frac{1}{\left|B_{1}\right|} 1_{B_{i}} D \phi_{i}\right): D\left(u_{g}\right) \\
& -2 \lambda^{2}\left\langle\left(\mu_{2} \delta_{x=y}-\bar{\mu}_{2, n}\right), D(\phi)(y) \otimes D\left(u_{g}\right)(x)\right\rangle=:-5 \lambda R_{1}+2 \lambda^{2} R_{2},
\end{aligned}
$$

with the notation that for $M$ a distribution over $\mathbb{R}^{3} \times \mathbb{R}^{3}$ with values in $\operatorname{Sym}\left(\operatorname{Sym}_{3, \sigma}(\mathbb{R})\right)$,

$$
\left\langle M, S \otimes S^{\prime}\right\rangle:=\left\langle M_{i j k l}, S_{i j} \otimes S_{k l}^{\prime}\right\rangle
$$

From assumption (A0), it easily follows that

$$
\lim _{n} R_{1}=0
$$

Then we write

$$
R_{2}=\left\langle\left(\mu_{2} \delta_{x=y}-\mu_{2, n}\right), D(\phi)(y) \otimes D\left(u_{g}\right)(x)\right\rangle+\widetilde{R}_{2},
$$

with

$$
\widetilde{R}_{2}=\left\langle\left(\mu_{2, n}-\bar{\mu}_{2, n}\right), D(\phi)(y) \otimes D\left(u_{g}\right)(x)\right\rangle .
$$

The first term on the right-hand side goes to zero as $n \rightarrow+\infty$, by assumption (A2). For the remainder, we decompose it as

$$
\begin{aligned}
\widetilde{R}_{2}= & \frac{75}{16 \pi n^{2}} \sum_{i \neq j}\left(\left(f_{B_{i}} \mathcal{M}\left(\cdot-x_{j}\right)\right)-\mathcal{M}\left(x_{i}-x_{j}\right)\right) D \phi_{j}:\left(f_{B_{i}} D\left(u_{g}\right)\right) \\
& +\frac{75}{16 \pi n^{2}} \sum_{i \neq j} \mathcal{M}\left(x_{i}-x_{j}\right) D \phi_{j}:\left(\left(f_{B_{i}} D\left(u_{g}\right)\right)-D\left(u_{g}\right)\left(x_{i}\right)\right) \\
& -\frac{75}{16 \pi} \int_{\mathbb{R}^{3}}(\mathcal{M} \star(D(\phi) \rho))(x)\left(\frac{1}{n} \sum_{i} \frac{1}{\left|B_{i}\right|}\left(f_{B_{i}} D\left(u_{g}\right)\right) 1_{B_{i}}-D\left(u_{g}\right) \rho\right)(x) d x \\
= & : \widetilde{R}_{2,1}+\widetilde{R}_{2,2}+\widetilde{R}_{2,3} .
\end{aligned}
$$

The first term is bounded by

$$
\left|\widetilde{R}_{2,1}\right| \leq \frac{75}{16 \pi n^{2}} \sum_{i \neq j} \sup _{z \in B_{i}}\left|\nabla \mathcal{M}\left(z-x_{j}\right)\right| r_{n}\left|D \phi_{j}\right|\left\|D\left(u_{g}\right)\right\|_{L^{\infty}(K)}
$$




$$
\begin{aligned}
& \leq \frac{C}{n^{2}} \sum_{i \neq j} \frac{r_{n}}{\left|x_{i}-x_{j}\right|^{4}}\left|D \phi_{j}\right| \leq \frac{C \lambda^{\frac{1}{3}}}{n} \sum_{i \neq j} \frac{1}{\left(c+\left|y_{i}-y_{j}\right|\right)^{4}}\left|D \phi_{j}\right| \\
& \leq \frac{C \lambda^{\frac{1}{3}}}{n} \sum_{i}\left|D \phi_{i}\right|,
\end{aligned}
$$

where the last line follows from (43). By (A0),

$$
\limsup _{n}\left|\widetilde{R}_{2,1}\right| \leq C \lambda^{\frac{1}{3}} \int_{\mathbb{R}^{3}}|D(\phi)| \rho \leq C \lambda^{\frac{1}{3}}\|D(\phi)\|_{L^{q}} \quad \forall q \geq 1 .
$$

Note that the constant $C$ may be chosen so that it depends on $g$ only through $\|g\|_{L^{3+\varepsilon}}$, using (48). The second term is bounded by

$$
\begin{aligned}
\left|\widetilde{R}_{2,2}\right| & \leq \frac{75}{16 \pi n^{2}} \sum_{i}\left|\sum_{j \neq i} \mathcal{M}\left(x_{i}-x_{j}\right) D \phi_{j}\right| r_{n}\left\|\nabla^{2} u_{g}\right\|_{L^{\infty}} \\
& \leq \frac{C}{n^{2} r_{n}^{2}} \sum_{i}\left|\sum_{j \neq i} r_{n}^{3} \mathcal{M}\left(x_{i}-x_{j}\right) D \phi_{j}\right| \leq \frac{C}{n^{\frac{3}{2}} r_{n}^{2}}\left(\sum_{i}\left|\sum_{j \neq i} r_{n}^{3} \mathcal{M}\left(x_{i}-x_{j}\right) D \phi_{j}\right|^{2}\right)^{1 / 2} \\
& \leq \frac{C}{n r_{n}^{2}}\left(\frac{1}{n} \sum_{i}\left|D \phi_{i}\right|^{2}\right)^{1 / 2} \leq \frac{C_{\lambda}}{n^{\frac{1}{3}}}\left(\int_{\mathbb{R}^{3}}|D(\phi)|^{2} \rho\right)^{1 / 2}
\end{aligned}
$$

where the fourth (resp. fifth) inequality is a consequence of Lemma 3.3 (resp. (A0)). Hence,

$$
\limsup _{n}\left|\widetilde{R}_{2,2}\right|=0
$$

As regards the last term $\widetilde{R}_{2,3}$, we use the fact that, under (A0),

$$
\frac{1}{n} \sum_{i} \frac{1}{\left|B_{i}\right|}\left(f_{B_{i}} D\left(u_{g}\right)\right) 1_{B_{i}} \underset{n \rightarrow+\infty}{\stackrel{D}{\longrightarrow}}\left(u_{g}\right) \rho \quad \text { weakly* in } L^{1} .
$$

See [9, Lem. 5] for a proof. Hence,

$$
\limsup _{n}\left|\widetilde{R}_{2,3}\right|=0
$$

Proposition 3.4 follows.

\section{Discussion of the mean-field limit}

In this final section we come back to the main assumption (A2): we discuss examples of particle distributions $\left(x_{i}\right)$ for which $\mu_{2, n}$ converges as in (A2), and discuss how to compute the limit $\mu_{2}$, that is, the $O\left(\lambda^{2}\right)$ correction to the effective viscosity. This discussion is closely related to the analysis performed in [8], and relies on results established there.

First, one notices that the sequence of compactly supported distributions $\mu_{2, n}$ defined in (6) is bounded with respect to $n$. More precisely, we have the following lemma: 
Lemma 4.1. There exists $C>0$, such that for any smooth $F=F(x, y)$, for any $n$,

$$
\left|\left\langle\mu_{2, n}, F\right\rangle\right| \leq C\|F\|_{C^{1}(K \times K)},
$$

where $K$ is any fixed convex compact set containing $x_{1}, \ldots, x_{n}$ for all $n$.

Proof. We need to bound

$$
\begin{aligned}
\frac{1}{n^{2}} \sum_{i \neq j} \mathcal{M}\left(x_{i}-x_{j}\right) F\left(x_{i}, x_{j}\right)= & \frac{1}{n^{2}} \sum_{i \neq j} \mathcal{M}\left(x_{i}-x_{j}\right) F\left(x_{j}, x_{j}\right) \\
& +\frac{1}{n^{2}} \sum_{i \neq j} \mathcal{M}\left(x_{i}-x_{j}\right)\left(F\left(x_{i}, x_{j}\right)-F\left(x_{j}, x_{j}\right)\right) \\
= & : I_{1, n}+I_{2, n} .
\end{aligned}
$$

As $\mathcal{M}$ is homogeneous of degree -3 and $\left|F\left(x_{i}, x_{j}\right)-F\left(x_{j}, x_{j}\right)\right| \leq\|\nabla F\|_{L^{\infty}(K \times K)} \times$ $\left|x_{i}-x_{j}\right|$,

$$
\left|I_{2, n}\right| \leq \frac{C}{n^{2}}\|\nabla F\|_{L^{\infty}} \sum_{i \neq j} \frac{1}{\left|x_{i}-x_{j}\right|^{2}} .
$$

We introduce $y_{i}:=x_{i} n^{-1 / 3}$ again, so that $\left|y_{i}-y_{j}\right| \geq \frac{1}{2}\left(c+\left|y_{i}-y_{j}\right|\right)$ with $c$ the constant appearing in (A1). We obtain

$$
\left|I_{2, n}\right| \leq \frac{C}{n^{2}} n^{\frac{2}{3}}\|\nabla F\|_{L^{\infty}} \sum_{i, j} \frac{1}{\left(c+\left|y_{i}-y_{j}\right|\right)^{2}} \leq C\|\nabla F\|_{L^{\infty}}
$$

using that for each $i$, under assumption (A1),

$$
\sum_{j} \frac{1}{\left(c+\left|y_{i}-y_{j}\right|\right)^{2}} \leq C \int_{n^{1 / 3} K} \frac{1}{\left(c+\left|y_{i}-z\right|\right)^{2}} d z \leq C n^{\frac{1}{3}} .
$$

To bound $I_{1, n}$, we introduce $s_{n}:=c /\left(4 n^{\frac{1}{3}}\right)$, where $c$ is again the constant appearing in (A1). We use the splitting

$$
\begin{aligned}
I_{1, n}= & \frac{1}{n^{2}} \sum_{i \neq j}\left(\mathcal{M}\left(x_{i}-x_{j}\right)-f_{B\left(x_{j}, s_{n}\right)} \mathcal{M}\left(x_{i}-y\right) d y\right) F\left(x_{j}, x_{j}\right) \\
& +\frac{1}{n^{2}} \sum_{i \neq j}\left(f_{B\left(x_{j}, s_{n}\right)} \mathcal{M}\left(x_{i}-y\right) d y-f_{B\left(x_{i}, s_{n}\right)} f_{B\left(x_{j}, s_{n}\right)} \mathcal{M}(x-y) d x d y\right) F\left(x_{j}, x_{j}\right) \\
& +\frac{1}{n^{2}} \sum_{i \neq j} f_{B\left(x_{i}, s_{n}\right)} f_{B\left(x_{j}, s_{n}\right)} \mathcal{M}(x-y) d x d y F\left(x_{j}, x_{j}\right)=: J_{1, n}+J_{2, n}+J_{3, n} .
\end{aligned}
$$

We find

$$
\left|J_{1, n}\right| \leq \frac{C}{n^{2}} \sum_{i \neq j} \sup _{z \in B\left(x_{j}, s_{n}\right)}\left|\nabla M\left(x_{i}-\cdot\right)\right| s_{n}\|F\|_{L^{\infty}}
$$




$$
\begin{aligned}
& \leq \frac{C s_{n}}{n^{2}} \sum_{i \neq j} \frac{1}{\left|x_{i}-x_{j}\right|^{4}}\|F\|_{L^{\infty}} \\
& \leq \frac{C}{n} \sum_{i \neq j} \frac{1}{\left(c+\left|y_{i}-y_{j}\right|\right)^{4}}\|F\|_{L^{\infty}} \leq C\|F\|_{L^{\infty}} .
\end{aligned}
$$

Similarly, we find $\left|J_{2, n}\right| \leq C$. Then, let $\chi$ be a truncation that is 0 on $B(0,1)$ and 1 outside $B(0,2)$. The last term can be written

$$
J_{3, n}=\frac{1}{n^{2}} \int_{\mathbb{R}^{3}}\left(\left(\chi\left(\frac{\dot{\bar{s}}}{s_{n}}\right) \mathcal{M}\right) \star \sum_{i} 1_{B\left(x_{i}, s_{n}\right)}\right) \sum_{j} F\left(x_{j}, x_{j}\right) 1_{B\left(x_{j}, s_{n}\right)}
$$

so that

$$
\left|J_{3, n}\right| \leq \frac{C}{n^{2}}\left\|\sum_{i} 1_{B\left(x_{i}, s_{n}\right)}\right\|_{L^{2}}\left\|\sum_{j} F\left(x_{j}, x_{j}\right) 1_{B\left(x_{j}, s_{n}\right)}\right\|_{L^{2}} \leq C\|F\|_{L^{\infty}}
$$

using the Calderon-Zygmund theorem. This concludes the proof.

Combining the previous lemma with the density of $\operatorname{span}\left(\left\{\varphi \otimes \tilde{\varphi}, \varphi, \tilde{\varphi} \in C^{\infty}(K)\right\}\right)$ in $C^{\infty}(K \times K)$, we deduce that for $\mu_{2} \in L^{\infty}\left(\mathbb{R}^{3}, \operatorname{Sym}\left(\operatorname{Sym}_{3, \sigma}(\mathbb{R})\right)\right)$, assumption (A2) is satisfied if and only if for all smooth $\varphi, \tilde{\varphi}$,

$$
\left\langle\mu_{2, n}, \varphi \otimes \tilde{\varphi}\right\rangle \rightarrow \int_{\mathbb{R}^{3}} \mu_{2}(x) \varphi(x) \tilde{\varphi}(x) d x
$$

or, equivalently, if and only if for all $S \in \operatorname{Sym}_{3, \sigma}(\mathbb{R})$, for all smooth $\varphi, \tilde{\varphi}$,

$$
\begin{aligned}
& \frac{75 \mu}{16 \pi}\left(\frac{1}{n^{2}} \sum_{i \neq j} g_{S}\left(x_{i}-x_{j}\right) \varphi\left(x_{i}\right) \tilde{\varphi}\left(x_{j}\right)-\int_{\mathbb{R}^{3}} g_{S}(x-y) \varphi(x) \tilde{\varphi}(y) d x d y\right) \\
& \quad \rightarrow \int_{\mathbb{R}^{3}}\left(\mu_{2}(x) S: S\right) \varphi(x) \tilde{\varphi}(x) d x,
\end{aligned}
$$

where

$$
g_{S}(x)=\mathcal{M}(x) S: S ;
$$

cf. (5). Since $g_{S}$ is even, this amounts to the following: for all $S \in \operatorname{Sym}_{3, \sigma}(\mathbb{R})$, for all smooth $\varphi$,

$$
\begin{aligned}
& \frac{75 \mu}{16 \pi}\left(\sum_{i \neq j} g_{S}\left(x_{i}-x_{j}\right) \varphi\left(x_{i}\right) \varphi\left(x_{j}\right)-\int_{\mathbb{R}^{3}} g_{S}(x-y) \varphi(x) \varphi(y) d x d y\right) \\
& \quad \rightarrow \int_{\mathbb{R}^{3}}\left(\mu_{2}(x) S: S\right)|\varphi(x)|^{2} d x .
\end{aligned}
$$

Of course, the existence of a nontrivial limit on the right-hand side of (51) is directly related to the singularity of $g_{s}$ at zero. If $g_{s}$ were smooth or not too singular, one could show that the limit on the right-hand side is zero. 
Our first goal is to understand configurations of points $\left(x_{i}\right)$ for which the limit as $n \rightarrow+\infty$ of $\frac{1}{n^{2}} \sum_{i \neq j} g_{S}\left(x_{i}-x_{j}\right) \varphi\left(x_{i}\right) \varphi\left(x_{j}\right)$ exists. This is closely related to the study in [8], in which the special case $\varphi=1$ is investigated. This study relies on ideas from homogenization theory, together with the notion of renormalized energy, described in [17] in the context of Coulomb gases. We shall explain how the approach in [8] adapts to a general $\varphi$. One proceeds in several steps.

\section{Step 1. Expression of the mean field as a (renormalized) energy}

Proceeding as in $[8$, Lem. 3.1], one can restrict to the case where

$$
x_{i} \in \mathcal{O} \quad \forall 1 \leq i \leq n .
$$

Under this assumption, proceeding as in [8, Prop. 3.3], one shows that (51), and thus (A2), amounts to the following: for all smooth $\varphi$, for all $S \in \operatorname{Sym}_{3, \sigma}(\mathbb{R})$,

$$
\lim _{n} \cdot \mu \mathcal{W}_{n}[\varphi]=\int_{\mathbb{R}^{3}}\left(\mu_{2}(x) S: S\right)|\varphi(x)|^{2} d x,
$$

where

$$
\mathcal{W}_{n}[\varphi]:=\frac{75}{16 \pi} \int_{\mathbb{R}^{6} \backslash \operatorname{Diag}} g_{S}(x-y) \varphi(x)\left(\rho_{n}(d x)-\rho(x) d x\right) \varphi(y)\left(\rho_{n}(d y)-\rho(y) d y\right) .
$$

To investigate the convergence of $\mathcal{W}_{n}[\varphi]$, the main idea is then to associate to this quadratic quantity an energy. This idea is based on the following lemma:

Lemma 4.2 ([8, Lem. 2.2]). For any $f \in L^{2}\left(\mathbb{R}^{3}\right)$,

$$
\int_{\mathbb{R}^{6}} g_{S}(x-y) f(x) f(y) d x d y=-\frac{16 \pi}{3} \int_{\mathbb{R}^{3}}\left|D\left(u_{S}\right)\right|^{2},
$$

where $u_{S}$ is the solution of the Stokes equation

$$
-\Delta u_{S}+\nabla q_{S}=\operatorname{div}(S f)=S \nabla f, \quad \operatorname{div} u_{S}=0 \quad \text { in } \mathbb{R}^{3} .
$$

Considering definition (53), it is tempting to replace $f$ by $\varphi\left(\rho_{n}-\rho\right)$ in the lemma, which would yield the formal identity

$$
\begin{gathered}
\int_{\mathbb{R}^{6}} g_{S}(x-y) \varphi(x)\left(\rho_{n}(d x)-\rho(x) d x\right) \varphi(y)\left(\rho_{n}(d y)-\rho(y) d y\right) \\
=-\frac{16 \pi}{3 n^{2}} \int_{\mathbb{R}^{3}}\left|D\left(h_{n}\right)\right|^{2},
\end{gathered}
$$

where $h_{n}$ solves the equation

$$
-\Delta h_{n}+\nabla p_{n}=n \operatorname{div}\left(S \varphi\left(\rho_{n}-\rho\right)\right), \quad \operatorname{div} h_{n}=0 \quad \text { in } \mathbb{R}^{3} .
$$

The solution of this equation decaying to zero is explicit, and given by

$$
h_{n}:=\sum \varphi\left(x_{i}\right) G_{S}\left(x-x_{i}\right)-n U \star S \nabla(\varphi \rho),
$$


where $G_{S}(x)=(S \nabla) \cdot \mathcal{U}(x)=-\frac{3}{8 \pi}(S x \cdot x) \frac{x}{|x|^{5}}$, while the corresponding pressure field is

$$
p_{n}=\sum \varphi\left(x_{i}\right) p_{S}\left(x-x_{i}\right)-n \mathcal{P} \star S \nabla(\varphi \rho),
$$

where $p_{S}(x)=(S \nabla) \cdot \mathcal{P}(x)=-\frac{3}{4 \pi} \frac{(S x \cdot x)}{|x|^{5}}$.

However, this formal identity is not justified, as both sides are infinite: the left-hand side is infinite due to the singularity of the kernel at the diagonal (which is excluded in definition (53)), and the right-hand side is infinite because the solution $h_{n}$ of (55) is not in $\dot{H}^{1}\left(\mathbb{R}^{3}\right)$, as $\rho_{n}$ is an atomic measure. Still, one can interpret $\mathcal{W}_{n}[\varphi]$ in terms of a so-called renormalized energy, intensively studied in the context of Coulomb gases and GinzburgLandau systems. See [17] and the references therein for more. One must first regularize the singular measure. More precisely, following [8], we consider for all $\eta>0$, the field $G_{S}^{\eta}$ satisfying

$$
G_{S}^{\eta}=G_{S}, \quad|x| \geq \eta, \quad-\Delta G_{S}^{\eta}+\nabla p_{S}^{\eta}=0, \quad \operatorname{div} G_{S}^{\eta}=0, \quad|x|<\eta,
$$

Note that the condition $G_{S}^{\eta}=G_{S}$ at $|x|=\eta$ contained in the first condition can be seen as a Dirichlet condition for the Stokes problem satisfied by $G_{S}^{\eta}$ inside $\{|x|<\eta\}$. Let us stress that $G_{S}^{\eta}$ belongs to $H_{\text {loc }}^{1}\left(\mathbb{R}^{3}\right)$, and can be seen as a regularization of $G_{S}$. Accordingly, we set

$$
h_{n}^{\eta}:=\sum_{i} \varphi\left(x_{i}\right) G_{S}^{\eta}\left(x-x_{i}\right)-n U \star S \nabla(\varphi \rho) .
$$

As shown in [8, Lem. 3.5], it solves the modified Stokes equation

$$
-\Delta h_{n}^{\eta}+\nabla p_{n}^{\eta}=\sum_{i} \varphi\left(x_{i}\right) \operatorname{div}\left(\Psi^{\eta}\left(\cdot-x_{i}\right)\right)-n \operatorname{div}(S \varphi \rho), \quad \operatorname{div} h_{n}^{\eta}=0 \quad \text { in } \mathbb{R}^{3},
$$

where

$$
\Psi^{\eta}:= \begin{cases}\frac{3}{\pi \eta^{5}}\left(S x \otimes x+x \otimes S x-5 \frac{|x|^{2}}{2} S+\frac{5}{4} \eta^{2} S\right) & \\ -2 D\left(G_{S}^{\eta}\right)(x)+p_{S}^{\eta}(x) \mathrm{Id}, & |x|<\eta, \\ 0, & |x|>\eta .\end{cases}
$$

A main advantage of this regularization is that $h_{n}-h_{n}^{\eta}$ is supported in $\bigcup_{i} B\left(x_{i}, \eta\right)$. The main result, which is a straightforward adaptation of [8, Props. 3.7 and 3.8] (dedicated to the special case $\varphi=1$ ) is given by the following proposition:

Proposition 4.3 (Renormalized energy formula). We have

$$
W_{n}[\varphi]=-\frac{25}{2} \lim _{\eta \rightarrow 0}\left(\frac{1}{n^{2}} \int_{\mathbb{R}^{3}}\left|\nabla h_{n}^{\eta}\right|^{2}-\frac{1}{n^{2} \eta^{3}}\left(\int_{B(0,1)}\left|\nabla G_{S}^{1}\right|^{2}+\frac{3}{10 \pi}|S|^{2}\right) \sum_{i}\left|\varphi\left(x_{i}\right)\right|^{2}\right) .
$$

More precisely, for $\eta<\frac{c}{2} n^{-\frac{1}{3}}$, where $c$ is the constant appearing in (A1), one has

$$
\left|\mathcal{W}_{n}[\varphi]+\frac{25}{2}\left(\frac{1}{n^{2}} \int_{\mathbb{R}^{3}}\left|\nabla h_{n}^{\eta}\right|^{2}-\frac{1}{n^{2} \eta^{3}}\left(\int_{B(0,1)}\left|\nabla G_{S}^{1}\right|^{2}+\frac{3}{10 \pi}|S|^{2}\right) \sum_{i}\left|\varphi\left(x_{i}\right)\right|^{2}\right)\right| \leq C \eta .
$$


The first formula in this proposition is the rigorous translation of the false identity (54): it connects the mean field $\mathcal{W}_{n}[\varphi]$ to an energy. However, two differences are apparent. First, the energy of $h_{n}$ being infinite, it is replaced by the energy of the regularization $h_{n}^{\eta}$. Second, and more importantly, one must subtract the term

$$
\frac{1}{\eta^{3}}\left(\int_{B(0,1)}\left|\nabla G_{S}^{1}\right|^{2}+\frac{3}{10 \pi}|S|^{2}\right) \sum_{i}\left|\varphi\left(x_{i}\right)\right|^{2},
$$

which corresponds to the energy of self-interaction, and blows up as $\eta \rightarrow 0$. It is only the difference of the two quantities that does not blow up as $\eta \rightarrow 0$ and gives $\mathcal{W}_{n}[\varphi]$. Note that the removal of the self-interaction is coherent with the fact that the expression for $\mathcal{W}_{n}[\varphi]$ excludes the diagonal; see (53).

\section{Step 2. Existence of the mean-field limit for periodic and random particle configurations}

Thanks to the second formula in Proposition 4.3, we deduce that

$$
\begin{aligned}
\lim _{n \rightarrow+\infty} \mathcal{W}_{n}[\varphi]= & -\frac{25}{2} \lim _{n \rightarrow+\infty} \frac{1}{n^{2}} \int_{\mathbb{R}^{3}}\left|\nabla h_{n}^{\eta_{n}}\right|^{2} \\
& -\frac{1}{n^{2} \eta_{n}^{3}}\left(\int_{B(0,1)}\left|\nabla G_{S}^{1}\right|^{2}+\frac{3}{10 \pi}|S|^{2}\right) \sum_{i}\left|\varphi\left(x_{i}\right)\right|^{2},
\end{aligned}
$$

when $\eta_{n}=\tilde{\eta} n^{-\frac{1}{3}}$, for any fixed $\tilde{\eta}<\frac{c}{2}$. Note that for such a scaling $\eta \sim n^{-\frac{1}{3}}$, contrary to the case where $\eta$ goes to zero at fixed $n$, the second term has a finite limit: namely, by (A0),

$$
\begin{aligned}
\lim _{n} & \frac{1}{n^{2} \eta^{3}}\left(\int_{B(0,1)}\left|\nabla G_{S}^{1}\right|^{2}+\frac{3}{10 \pi}|S|^{2}\right) \sum_{i}\left|\varphi\left(x_{i}\right)\right|^{2} \\
= & \frac{1}{(\tilde{\eta})^{3}}\left(\int_{B(0,1)}\left|\nabla G_{S}^{1}\right|^{2}+\frac{3}{10 \pi}|S|^{2}\right) \int_{\mathbb{R}^{3}}|\varphi|^{2} \rho .
\end{aligned}
$$

Hence, the main point is to understand the limit of $\frac{1}{n^{2}} \int_{\mathbb{R}^{3}}\left|\nabla h_{n}^{\eta_{n}}\right|^{2}$. In the case $\varphi=1$, this is analyzed in detail in [8], for two classes of point configurations: periodic and random stationary. Namely, in [8] one considers particles $x_{1}, \ldots, x_{n}$ given by

$$
\left\{x_{1}, \ldots, x_{n}\right\}:=\varepsilon \omega \cap \mathcal{O}, \quad \varepsilon \ll 1
$$

for $\omega$ an infinite locally finite subset of $\mathbb{R}^{3}$, of two possible kinds:

(i) Periodic patterns: $\omega=\left\{z_{1}, \ldots, z_{m}\right\}+\mathbb{Z}^{3}$ for $z_{1}, \ldots, z_{m}$ distinct points of $(0,1)^{3}$, such that $\left|z-z^{\prime}\right|>c m^{-\frac{1}{3}}$ for all $z \neq z^{\prime} \in \omega$, where $c$ is the constant in (A1).

(ii) Stationary point process: $\omega$ is the realization of an ergodic stationary point process, of mean intensity $m$, satisfying the hardcore condition $\left|z-z^{\prime}\right|>\mathrm{cm}^{-\frac{1}{3}}$ for all $z \neq z^{\prime} \in \omega$. 
Note that in the periodic case, the number $n$ of particles in $\mathcal{O}$ is equivalent to $m \varepsilon^{-3}$ as $\varepsilon \rightarrow 0$. In the random case, this number $n$ is random, but is almost surely still equivalent to $m \varepsilon^{-3}$ by the ergodic theorem (under an additional convexity assumption on $\mathcal{O}$ ). In particular, as $\varepsilon \rightarrow 0$, one has $n \rightarrow+\infty$ and one can consider the asymptotic behaviour of $\mathcal{W}_{n}[\varphi]$ or, as said before, of $\frac{1}{n^{2}} \int_{\mathbb{R}^{3}}\left|\nabla h_{n}^{\eta_{n}}\right|^{2}$. Note that in both cases, assumption (A0) is satisfied with $\rho=1_{\mathcal{O}}$, and for $\varepsilon$ small enough (or $n$ large enough), (A1) is also satisfied.

The limit of the renormalized energy can then be tackled through homogenization techniques. Indeed, we claim that equation (58) is close to systems of the form

$$
-\Delta h^{\varepsilon}+\nabla p^{\varepsilon}=\varepsilon^{-3} \operatorname{div}(f(x)(F(x / \varepsilon)-\bar{F})), \quad \operatorname{div} h^{\varepsilon}=0,
$$

where $\bar{F}$ is the mean of $F$ (in the periodic or random sense). Roughly, one has the correspondence

$$
\varepsilon \approx n^{-\frac{1}{3}}, \quad F \approx \sum_{z \in \omega} \Psi^{\tilde{\eta}}(\cdot-z), \quad \bar{F} \approx 1, \quad f \approx 1_{\mathcal{O}} \varphi
$$

The point is that for a model of type (60), the behaviour of the solution $h^{\varepsilon}$ is well understood. For instance, in the periodic case one has

$$
h^{\varepsilon} \approx \varepsilon^{-2} g(x) H(x / \varepsilon),
$$

where $H=H(y)$ is a corrector, solving

$$
-\Delta H+\nabla P=\operatorname{div} F, \quad \operatorname{div} H=0,
$$

with periodic boundary conditions. In particular, one can show that

$$
\varepsilon^{6} \int_{\mathbb{R}^{3}}\left|\nabla h^{\varepsilon}\right|^{2} \rightarrow \int_{(0,1)^{3}}|\nabla H(y)|^{2} d y \int_{\mathbb{R}^{3}}|g(x)|^{2} d x .
$$

As shown in [8] in the special case $\varphi=1$, a similar situation holds in the context of system (58). There is a corrector problem similar to (62), that takes the form

$$
-\Delta H^{\bar{\eta}}+\nabla P^{\bar{\eta}}=\operatorname{div} \sum_{z \in \omega} \Psi^{\bar{\eta}}(\cdot-z), \quad \operatorname{div} H^{\bar{\eta}}=0 \quad \text { in } \mathbb{R}^{3},
$$

where $0<\bar{\eta}<\frac{c}{2} m^{-\frac{1}{3}}$. This can be solved in both periodic and random stationary frameworks, as shown in [8]. Roughly,

(i) in the periodic case, there is a unique (up to an additive constant) weak solution $H^{\bar{\eta}}$ in $H_{\mathrm{loc}}^{1}\left(\mathbb{R}^{3}\right)$, which is $\mathbb{Z}^{d}$ periodic and mean-free over a period;

(ii) in the random stationary case, there is almost surely in $\omega$ a weak solution $H^{\bar{\eta}}(\cdot, \omega)$ in $H_{\text {loc }}^{1}\left(\mathbb{R}^{3}\right)$ with a uniquely defined stationary and mean-free gradient. More precisely, $\nabla H^{\bar{\eta}}(y, \omega)=D_{H}(\omega-y)$, where $D_{H}$ is an $\mathbb{R}^{3}$-valued $L^{2}$ random variable with zero mean, the unique solution of a probabilistic variational formulation; see [8] for details.

One can then express our mean field limit in terms of the energy of this corrector. This is the following proposition: 
Proposition 4.4. Let $\bar{\eta}<\frac{c}{2} m^{-\frac{1}{3}}$. Then, as $\varepsilon \rightarrow 0$, one has $n \rightarrow+\infty$, and

$$
\mathcal{W}_{n}[\varphi] \rightarrow \frac{25}{2}\left(-\frac{1}{m^{2}} \int_{(0,1)^{3}}\left|\nabla H^{\bar{\eta}}\right|^{2}+\frac{1}{(\bar{\eta})^{3} m}\left(\int_{B(0,1)}\left|\nabla G_{S}^{1}\right|^{2}+\frac{3}{10 \pi}|S|^{2}\right)\right) \int_{\mathcal{O}}|\varphi|^{2}
$$

in the periodic case, whereas

$$
\mathcal{W}_{n}[\varphi] \rightarrow \frac{25}{2}\left(-\frac{1}{m^{2}} \mathbb{E}\left|\nabla H^{\bar{\eta}}(0, \cdot)\right|^{2}+\frac{1}{(\bar{\eta})^{3} m}\left(\int_{B(0,1)}\left|\nabla G_{S}^{1}\right|^{2}+\frac{3}{10 \pi}|S|^{2}\right)\right) \int_{\mathcal{O}}|\varphi|^{2}
$$

in the random stationary case. In particular, assumption (A2) is satisfied with

$$
\mu_{2} S: S=\frac{25 \mu}{2}\left(-\frac{1}{m^{2}} \int_{(0,1)^{3}}\left|\nabla H^{\bar{\eta}}\right|^{2}+\frac{1}{(\bar{\eta})^{3} m}\left(\int_{B(0,1)}\left|\nabla G_{S}^{1}\right|^{2}+\frac{3}{10 \pi}|S|^{2}\right)\right)
$$

in the periodic case, whereas

$$
\mu_{2} S: S=\frac{25 \mu}{2}\left(-\frac{1}{m^{2}} \mathbb{E}\left|\nabla H^{\bar{\eta}}(0, \cdot)\right|^{2}+\frac{1}{(\bar{\eta})^{3} m}\left(\int_{B(0,1)}\left|\nabla G_{S}^{1}\right|^{2}+\frac{3}{10 \pi}|S|^{2}\right)\right)
$$

in the random stationary case.

This proposition was established in [8] in the case $\varphi=1$, and the proof extends without too much difficulty to the general case. Note that the right-hand sides that appear above do not actually depend on $\bar{\eta}$ (as long as $\bar{\eta}<\frac{c}{2} m^{-\frac{1}{3}}$ ), as the left-hand side $\mathcal{W}_{n}[\varphi]$ does not involve $\bar{\eta}$. See [8, Prop. 4.4] for a direct proof.

Let us briefly evoke the main steps of the proof of Proposition 4.4, restricting to the periodic case for brevity. Keeping in mind that $n \sim m \varepsilon^{-3}$ as $\varepsilon \rightarrow 0$, the point is to show that

$$
\varepsilon^{6} \int_{\mathbb{R}^{3}}\left|\nabla h^{\bar{\eta} \varepsilon}\right|^{2} \rightarrow \int_{(0,1)^{3}}\left|\nabla H^{\bar{\eta}}\right|^{2} \int_{\mathcal{O}}|\varphi|^{2} .
$$

The idea is to introduce an approximation of $h^{\bar{\eta} \varepsilon}$ using the corrector solution of (62). Namely, we introduce an approximate velocity field $h_{\text {app }}^{\varepsilon} \in H_{\text {loc }}^{1}\left(\mathbb{R}^{3}\right)$ and pressure field $p_{\text {app }}^{\varepsilon} \in L_{\text {loc }}^{2}\left(\mathbb{R}^{3}\right)$ defined by

$$
\begin{aligned}
& h_{\mathrm{app}}^{\varepsilon}(x):=\frac{1}{\varepsilon^{2}} \varphi(x) H^{\bar{\eta}}\left(\frac{x}{\varepsilon}\right)-\int_{\mathcal{O}} \frac{1}{\varepsilon^{2}} \varphi(x) H^{\bar{\eta}}\left(\frac{x}{\varepsilon}\right) d x, \quad x \in \mathcal{O}, \\
& p_{\mathrm{app}}^{\varepsilon}(x):=\frac{1}{\varepsilon^{3}} \varphi(x) P^{\bar{\eta}}\left(\frac{x}{\varepsilon}\right)-\int_{\mathcal{O}} \frac{1}{\varepsilon^{3}} \varphi(x) P^{\bar{\eta}}\left(\frac{x}{\varepsilon}\right) d x, \quad x \in \mathcal{O},
\end{aligned}
$$

and

$$
-\Delta h_{\text {app }}^{\varepsilon}+\nabla p_{\text {app }}^{\varepsilon}=0, \quad \operatorname{div} h_{\text {app }}^{\varepsilon}=0, \quad \text { in } \mathbb{R}^{3} \backslash \overline{\mathcal{O}}
$$

One has straightforwardly

$$
\varepsilon^{6} \int_{\mathcal{O}}\left|\nabla h_{\mathrm{app}}^{\varepsilon}\right|^{2} \rightarrow \int_{(0,1)^{3}}\left|\nabla H^{\bar{\eta}}\right|^{2} \int_{\mathcal{O}}|\varphi|^{2}, \quad \varepsilon \rightarrow 0 .
$$


Moreover, reasoning exactly as in [8], one shows that the $H^{\frac{1}{2}}(\partial \mathcal{O})$ norm of $h_{\text {app }}^{\varepsilon}$ goes to zero as $\varepsilon \rightarrow 0$, which implies

$$
\left\|\nabla h_{\mathrm{app}}^{\varepsilon}\right\|_{L^{2}\left(\mathbb{R}^{3} \backslash \overline{\mathcal{O}}\right)} \rightarrow 0, \quad \varepsilon \rightarrow 0
$$

so that

$$
\varepsilon^{6} \int_{\mathbb{R}^{3}}\left|\nabla h_{\mathrm{app}}^{\varepsilon}\right|^{2} \rightarrow \int_{(0,1)^{3}}\left|\nabla H^{\bar{\eta}}\right|^{2} \int_{\mathcal{O}}|\varphi|^{2}, \quad \varepsilon \rightarrow 0 .
$$

The final step of the proof consists in showing that

$$
\varepsilon^{6} \int_{\mathbb{R}^{3}}\left|\nabla\left(h^{\bar{\eta} \varepsilon}-h_{\mathrm{app}}^{\varepsilon}\right)\right|^{2} \rightarrow 0, \quad \varepsilon \rightarrow 0 .
$$

This is a consequence of an energy estimate, performed on the Stokes equation satisfied by the difference $h^{\bar{\eta} \varepsilon}-h_{\text {app }}^{\varepsilon}$. In the case $\varphi=1$, all details are provided in [8]. For general $\varphi$, there are a few extra source terms in this Stokes equation, but they can be handled through similar ideas, so that we do not give further detail.

\section{Step 3. Explicit computation of $\mu_{2}$}

Finally, as further explained in [8], one can make formulas (64) and (65) more explicit. In the periodic case, with $\omega=\left\{z_{1}, \ldots, z_{m}\right\}+\mathbb{Z}^{3}$, the following holds.

Proposition 4.5 (Periodic case; see [8, Props. 5.4 and 5.5]).

(1) One has

$$
\begin{aligned}
\mu_{2} S: S=\frac{25 \mu}{2 m^{2}}( & \sum_{i \neq j \in\{1, \ldots, m\}} S \nabla \cdot G_{S, 1}\left(z_{i}-z_{j}\right) \\
& \left.+m \lim _{y \rightarrow 0} S \nabla \cdot\left(G_{S, 1}(y)-G_{S}(y)\right)\right),
\end{aligned}
$$

where $G_{S}(x)=(S \nabla) \cdot \mathcal{U}(x)=-\frac{3}{8 \pi}(S x \cdot x) \frac{x}{|x|^{5}}$ solves

$$
-\Delta G_{S}+\nabla p_{S}=(S \nabla) \delta, \quad \operatorname{div} G_{S}=0, \quad \text { in } \mathbb{R}^{3}
$$

and for all $L>0, G_{S, L}$ is the periodic field with zero mean solution of

$$
-\Delta G_{S, L}+\nabla p_{S, L}=(S \nabla) \sum_{z \in L \mathbb{Z}^{3}} \delta_{z}, \quad \operatorname{div} G_{S, L}=0, \quad \text { in } \mathbb{R}^{3} .
$$

(2) In the special case of a simple cubic lattice $(m=1)$, the formula simplifies to

$$
\mu_{2} S: S=\mu \alpha \sum_{i} S_{i i}^{2}+\mu \beta \sum_{i \neq j} S_{i j}^{2},
$$

with $\alpha=\frac{5}{2}(1-60 a), \beta=\frac{5}{2}(1+40 a)$, and $a \approx-0.04655$. 
In the ergodic stationary case, under the almost sure assumption (A1), a more explicit expression can be obtained in terms of the two-point correlation function $\rho_{2}(x, y)=$ $r(x-y)$. We recall that a point process in $\mathbb{R}^{3}$ has a two-point correlation function $\rho_{2} \in$ $L_{\text {loc }}^{1}\left(\mathbb{R}^{3} \times \mathbb{R}^{3}\right)$ if for all bounded $K$ and smooth functions $F$,

$$
\mathbb{E} \sum_{z \neq z^{\prime} \in K} F\left(z, z^{\prime}\right)=\int_{K \times K} F(x, y) \rho_{2}(x, y) d x d y .
$$

Proposition 4.6 (Ergodic stationary case; see [8, Prop. 5.6]). Assume that $\rho_{2}(x, y)=$ $r(x-y)$ with $r \in L_{\text {loc }}^{1}\left(\mathbb{R}^{3}\right)$, zero near the origin. Then, for any $M>0$, almost surely,

$\mu_{2} S: S=\frac{25 \mu}{2 m^{2}} \lim _{L \rightarrow+\infty} \frac{1}{L^{3}} \int_{(M, L-M)^{3} \times(M, L-M)^{3}}(S \nabla) \cdot G_{S, L}\left(z-z^{\prime}\right) r\left(z-z^{\prime}\right) d z d z^{\prime}$,

where $G_{S, L}$ was introduced in (66).

One can actually push this calculation further in the case of an isotropic process. The next proposition is the first justification of a formula that was derived formally in [18].

Proposition 4.7 (Isotropic point process). Assume that $\rho_{2}(x, y)=r(x-y)$ with $r$ zero near the origin, radial and such that $\left|r(x)-m^{2}\right| \leq \omega(|x|)$ for some bounded and decreasing function $\omega: \mathbb{R}_{+} \rightarrow \mathbb{R}_{+}$with $\int_{\mathbb{R}_{+}} \frac{\omega(t)}{t} d t<+\infty$. Then, almost surely,

$$
\mu_{2} S: S=\frac{5}{2} \mu|S|^{2}
$$

Remark 4.1. Let us note that the formula above $\left(\mu_{2}=\frac{5}{2}\right)$ differs from the one derived formally by Batchelor and Green ([2]) for isotropic random processes of Poisson type with a no-penetration condition $\left(\mu_{2} \approx 7.6\right)$. The reason is that in the case considered in [2], assumption (A1) is not satisfied. Roughly, it follows that the kernel $G_{S, L}\left(z-z^{\prime}\right)$ appearing in Proposition 4.6 must be modified: the appropriate kernel $G\left(z-z^{\prime}\right)$ is only close to $G_{S, L}$ asymptotically as $\left|z-z^{\prime}\right| \rightarrow+\infty$. This point was raised in [2, p. 418]. Since our paper was submitted for publication, rigorous results have been obtained on the Batchelor-Green formula; see [3,7].

Remark 4.2. The fact that $r$ is radial in the proposition corresponds to the isotropy of the point process. The weak decay condition on $r-m^{2}$ corresponds to a natural decorrelation at large distances. See [3] for similar assumptions. Note that all assumptions of the proposition are satisfied by the usual hardcore Poisson processes.

Proof of Proposition 4.7. For any $L>2 M \geq 0$, we denote $K_{M, L}:=(M, L-M)^{3}$. By Proposition 4.6, it is enough to prove that

$$
\lim _{M \rightarrow+\infty} \lim _{L \rightarrow+\infty} \frac{1}{L^{3}} \int_{K_{M, L} \times K_{M, L}}(S \nabla) \cdot G_{S, L}\left(z-z^{\prime}\right) r\left(z-z^{\prime}\right) d z d z^{\prime}=\frac{m^{2}}{5}|S|^{2} .
$$


By a simple scaling argument, $G_{S, L}(x)=L^{-2} G_{S, 1}\left(\frac{x}{L}\right)$. It follows easily that

$$
\begin{aligned}
& \frac{1}{L^{3}} \int_{K_{M, L} \times K_{M, L}}(S \nabla) \cdot G_{S, L}\left(z-z^{\prime}\right) r\left(z-z^{\prime}\right) d z d z^{\prime} \\
& \quad=\int_{K_{M / L, 1} \times K_{M / L, 1}}(S \nabla) \cdot G_{S, 1}\left(z-z^{\prime}\right) r\left(L\left(z-z^{\prime}\right)\right) d z d z^{\prime}
\end{aligned}
$$

For $z, z^{\prime} \in K_{M / L, 1}$, we have $z-z^{\prime} \in(-1+2 M / L, 1-2 M / L)^{3}$. Over this set, the periodic function $G_{S, 1}$ can be decomposed as

$$
G_{S, 1}=\sum_{k \in \mathbb{Z}^{3},\left|k_{i}\right| \leq 1} G_{S}(\cdot-k)+\widetilde{G}_{S, 1},
$$

where $\widetilde{G}_{S, 1}$ is smooth. From this decomposition, and the fact that $r(x)=0$ for all $|x| \leq \delta$, $\delta>0$ small enough, it follows that

$$
\begin{aligned}
& \int_{(-1+2 M / L, 1-2 M / L)^{3}}\left|(S \nabla) \cdot G_{S, 1}(u) r(L u)\right| d u \\
& \quad \leq C \int_{(-1+2 M / L, 1-2 M / L)^{3} \cap\{|u| \geq \delta / L\}}\left(\sum_{k \in \mathbb{Z}^{3},\left|k_{i}\right| \leq 1} \frac{1}{|u-k|^{3}}+1\right) d u \\
& \quad \leq C_{M} \ln (L) .
\end{aligned}
$$

It follows in particular that

$$
\begin{aligned}
& \int_{\left(K_{M / L, 1} \backslash K_{2 M / L, 1}\right) \times K_{M / L, 1}}(S \nabla) \cdot G_{S, 1}\left(z-z^{\prime}\right) r\left(L\left(z-z^{\prime}\right)\right) d z d z^{\prime} \\
& \leq\left|K_{M / L, 1} \backslash K_{2 M / L, 1}\right| C_{M} \ln L \leq C_{M}^{\prime} \frac{\ln L}{L} \rightarrow 0, \quad L \rightarrow+\infty .
\end{aligned}
$$

It remains to show that

$$
\lim _{M \rightarrow+\infty} \lim _{L \rightarrow+\infty} \int_{K_{2 M / L, 1} \times K_{M / L, 1}}(S \nabla) \cdot G_{S, 1}\left(z-z^{\prime}\right) r\left(L\left(z-z^{\prime}\right)\right) d z d z^{\prime}=\frac{m^{2}}{5}|S|^{2} .
$$

We decompose

$$
\begin{aligned}
& \int_{K_{2 M / L, 1} \times K_{M / L, 1}}(S \nabla) \cdot G_{S, 1}\left(z-z^{\prime}\right) r\left(L\left(z-z^{\prime}\right)\right) d z d z^{\prime} \\
&= \int_{K_{2 M / L, 1}} \int_{K_{M / L, 1} \cap B\left(z^{\prime}, M / L\right)^{c}}(S \nabla) \cdot G_{S, 1}\left(z-z^{\prime}\right) m^{2} d z d z^{\prime} \\
&+\int_{K_{2 M / L, 1}} \int_{K_{M / L, 1} \cap B\left(z^{\prime}, M / L\right)^{c}}(S \nabla) \cdot G_{S, 1}\left(z-z^{\prime}\right)\left(r\left(L\left(z-z^{\prime}\right)\right)-m^{2}\right) d z d z^{\prime} \\
&+\int_{K_{2 M / L, 1}} \int_{B\left(z^{\prime}, M / L\right)}(S \nabla) \cdot G_{S, 1}\left(z-z^{\prime}\right) r\left(L\left(z-z^{\prime}\right)\right) d z d z^{\prime} \\
&=: I_{M, L}^{1}+I_{M, L}^{2}+I_{M, L}^{3} .
\end{aligned}
$$


We have used that for any $z^{\prime} \in K_{2 M / L, 1}, K_{M / L, 1} \cap B\left(z^{\prime}, M / L\right)=B\left(z^{\prime}, M / L\right)$. As regards the first term, we use (68) again, which yields

$$
\int_{K_{2 M / L, 1}} \int_{K_{0,1} \backslash K_{M / L, 1}}\left|(S \nabla) \cdot G_{S, 1}\left(z-z^{\prime}\right) m^{2}\right| d z d z^{\prime} \leq C_{M} \frac{\ln L}{L},
$$

so that

$$
I_{M, L}^{1}=\int_{K_{2 M / L, 1}} \int_{K_{0,1} \cap B\left(z^{\prime}, M / L\right)^{c}}(S \nabla) \cdot G_{S, 1}\left(z-z^{\prime}\right) m^{2} d z d z^{\prime}+o(1),
$$

where the term $o(1)$ goes to zero as $L \rightarrow+\infty$ for any fixed $M$. We then perform an integration by parts in the variable $z$. Note that for all $z^{\prime} \in K_{2 M / L, 1}$, the boundary of the ball $B\left(z^{\prime}, M / L\right)$ is disjoint from the boundary of $K_{0,1}$. Taking into account that $(S \nabla) \cdot G_{S, 1}\left(z-z^{\prime}\right)$ is $\mathbb{Z}^{d}$-periodic, the boundary term at $\partial K_{0,1}$ vanishes, and eventually

$$
\begin{aligned}
I_{M, L}^{1} & =-\int_{K_{2 M / L, 1}} \int_{\partial B\left(z^{\prime}, M / L\right)}(S v) \cdot G_{S, 1}\left(z-z^{\prime}\right) d \sigma(z) d z^{\prime} m^{2}+o(1) \\
& =-\int_{K_{2 M / L, 1}} \int_{\partial B\left(z^{\prime}, M / L\right)}(S v) \cdot G_{S}\left(z-z^{\prime}\right) d \sigma(z) d z^{\prime} m^{2}+o(1) \\
& =-\left(1-\frac{4 M}{L}\right)^{3}\left(\int_{\partial B(0,1)}(S v) \cdot G_{S}\right) m^{2}+o(1)
\end{aligned}
$$

Using the explicit expression for $G_{S}$, we find that for any $M>0$,

$$
I_{M, L}^{1} \rightarrow \frac{3}{8 \pi} \int_{\partial B_{1}}(S n \cdot n)^{2} m^{2}=\frac{m^{2}}{5}|S|^{2} \quad \text { as } L \rightarrow+\infty,
$$

thanks to the identity $\int_{\partial B_{1}} n_{i} n_{j} n_{k} n_{l}=\frac{4 \pi}{15}\left(\partial_{i j} \partial_{k l}+\partial_{i k} \partial_{j l}+\partial_{i l} \partial_{j k}\right)$. As regards the second term, using decomposition (67), we find the upper bound

$$
\begin{aligned}
& \left|I_{M, L}^{2}\right| \\
& \quad \leq C \int_{\substack{(-1+2 M / L, 1-2 M / L)^{3} \\
\cap\{|u| \geq M / L\}}}\left(\sum_{k \in \mathbb{Z}^{3},\left|k_{i}\right| \leq 1} \frac{1}{|u-k|^{3}}+1\right)\left|r(L u)-m^{2}\right| d u \\
& \quad \leq C \sum_{k \in \mathbb{Z}^{3},\left|k_{i}\right| \leq 1} \int_{\substack{(-L+2 M, L-2 M)^{3} \\
\cap\left\{\left|u^{\prime}\right| \geq M\right\}}} \frac{\left|r\left(u^{\prime}\right)-m^{2}\right|}{\left|u^{\prime}-L k\right|^{3}} d u^{\prime}+C \sup _{\left|u^{\prime}\right| \geq M}\left|r\left(u^{\prime}\right)-m^{2}\right| .
\end{aligned}
$$

Clearly, the last term on the right-hand side is independent of $L$ and goes to zero when $M \rightarrow+\infty$. Moreover, we claim that all integrals on the right-hand side go to zero when $M \rightarrow+\infty$, uniformly in $L$. Indeed, for $k=0$, we simply write

$$
\int_{\substack{(-L+2 M, L-2 M)^{3} \\ \cap\left\{\left|u^{\prime}\right| \geq M\right\}}} \frac{\left|r\left(u^{\prime}\right)-m^{2}\right|}{\left|u^{\prime}\right|^{3}} d u^{\prime} \leq \int_{|t| \geq M} \frac{\omega(t)}{t} d t \rightarrow 0, \quad M \rightarrow+\infty .
$$


For $k \neq 0$ in the sum, we write

$$
\begin{aligned}
\int_{\substack{(-L+2 M, L-2 M)^{3} \\
\cap\left\{\left|u^{\prime}\right| \geq M\right\}}} \frac{\left|r\left(u^{\prime}\right)-m^{2}\right|}{\left|u^{\prime}-L k\right|^{3}} d u^{\prime} \leq & \int_{\substack{\left\{\sqrt{3} L \geq\left|u^{\prime}\right| \geq M\right\} \\
\cap\left\{\left|u^{\prime}-L k\right| \geq 2 M\right\}}} \frac{\omega\left(\left|u^{\prime}\right|\right)}{\left|u^{\prime}-L k\right|^{3}} d u^{\prime} \\
\leq & \int_{\substack{\left\{\sqrt{3} L \geq\left|u^{\prime}\right| \geq M\right\} \\
\cap\left\{\frac{1}{2} L \geq\left|u^{\prime}-L k\right| \geq 2 M\right\}}} \frac{\omega\left(\left|u^{\prime}\right|\right)}{\left|u^{\prime}-L k\right|^{3}} d u^{\prime} \\
& +\int_{\substack{\left\{\sqrt{3} L \geq\left|u^{\prime}\right| \geq M\right\} \\
\cap\left\{\left|u^{\prime}-L k\right| \geq \frac{1}{2} L\right\}}} \frac{\omega\left(\left|u^{\prime}\right|\right)}{\left|u^{\prime}-L k\right|^{3}} d u^{\prime} .
\end{aligned}
$$

For the second integral, we use the lower bound $\left|u^{\prime}-L k\right| \geq \frac{1}{2} L$ for the denominator, so that

$$
\int_{\substack{\left\{\sqrt{3} L \geq\left|u^{\prime}\right| \geq M\right\} \\ \cap\left\{\left|u^{\prime}-L k\right| \geq \frac{1}{2} L\right\}}} \frac{\omega\left(\left|u^{\prime}\right|\right)}{\left|u^{\prime}-L k\right|^{3}} d u^{\prime} \leq C \omega(M) .
$$

For the first integral, we notice that

$\left|u^{\prime}\right| \geq|L k|-\left|u^{\prime}-L k\right| \geq L-\frac{1}{2} L=\frac{1}{2} L \geq\left|u^{\prime}-L k\right|, \quad$ so that $\omega\left(\left|u^{\prime}\right|\right) \leq \omega\left(\left|u^{\prime}-L k\right|\right)$.

We infer

$$
\begin{aligned}
\int_{\substack{\left\{\sqrt{3} L \geq\left|u^{\prime}\right| \geq M\right\} \\
\cap\left\{\frac{1}{2} L \geq\left|u^{\prime}-L k\right| \geq 2 M\right\}}} \frac{\omega\left(\left|u^{\prime}\right|\right)}{\left|u^{\prime}-L k\right|^{3}} d u^{\prime} & \leq \int_{\left\{\frac{1}{2} L \geq\left|u^{\prime}-L k\right| \geq 2 M\right\}} \frac{\omega\left(\left|u^{\prime}-L k\right|\right)}{\left|u^{\prime}-L k\right|^{3}} \\
& \leq \int_{|t| \geq 2 M} \frac{\omega(t)}{t} d t \rightarrow 0, \quad M \rightarrow+\infty
\end{aligned}
$$

Hence, $\lim _{M \rightarrow+\infty} \lim \sup _{L \rightarrow+\infty}\left|I_{M, L}^{2}\right|=0$. Finally, for the last term, we find

$$
I_{M, L}^{3}=\int_{K_{2 M / L, 1}} \int_{B\left(z^{\prime}, M / L\right)}(S \nabla) \cdot G_{S}\left(z-z^{\prime}\right) r\left(L\left(z-z^{\prime}\right)\right) d z d z^{\prime}+o(1),
$$

where again the $o(1)$ refers to a quantity going to zero as $L \rightarrow+\infty$ for any fixed $M$. As $r$ is radial and as the mean of $(S \nabla) \cdot G_{S}$ over spheres is zero, the integral in $z$ on the right-hand side vanishes identically. This concludes the proof.

Funding. The authors acknowledge the support of the SingFlows project, grant ANR18-CE40-0027 of the French National Research Agency (ANR). D.G.-V. acknowledges the support of the Institut Universitaire de France. A.M. acknowledges funding from the European Research Council (ERC) under the European Union's Horizon 2020 research and innovation program grant agreement no. 637653, project BLOC "Mathematical Study of Boundary Layers in Oceanic Motion".

\section{References}

[1] H. Ammari, P. Garapon, H. Kang, and H. Lee, Effective viscosity properties of dilute suspensions of arbitrarily shaped particles. Asymptot. Anal. 80 (2012), no. 3-4, 189-211 Zbl 1341.76033 MR 3025042 
[2] G. K. Batchelor and J. T. Green, The determination of the bulk stress in a suspension of spherical particles to order $c^{2}$. J. Fluid Mech. 56 (1972), 401-427 Zbl 0246.76108

[3] M. Duerinckx and A. Gloria, On Einstein's effective viscosity formula. 2020, arXiv:2008.03837

[4] M. Duerinckx and A. Gloria, Corrector equations in fluid mechanics: effective viscosity of colloidal suspensions. Arch. Ration. Mech. Anal. 239 (2021), no. 2, 1025-1060 Zbl 1456.76134 MR 4201621

[5] A. Einstein, Eine neue Bestimmung der Moleküldimensionen. Ann. Phys. 19 (1906), 289-306 Zbl 37.0811 .01

[6] G. P. Galdi, An introduction to the mathematical theory of the Navier-Stokes equations. 2nd edn., Springer Monogr. Math., Springer, New York, 2011 Zbl 1245.35002 MR 2808162

[7] D. Gérard-Varet, Derivation of the Batchelor-Green formula for random suspensions. J. Math. Pures Appl. (9) 152 (2021), 211-250 Zbl 07379402 MR 4280836

[8] D. Gérard-Varet and M. Hillairet, Analysis of the viscosity of dilute suspensions beyond Einstein's formula. Arch. Ration. Mech. Anal. 238 (2020), no. 3, 1349-1411 Zbl 1454.76101 MR 4160802

[9] D. Gérard-Varet and R. M. Höfer, Mild assumptions for the derivation of Einstein's effective viscosity formula. Comm. Partial Differential Equations 46 (2021), no. 4, 611-629 Zbl 07378230 MR 4260456

[10] B. M. Haines and A. L. Mazzucato, A proof of Einstein's effective viscosity for a dilute suspension of spheres. SIAM J. Math. Anal. 44 (2012), no. 3, 2120-2145 Zbl 1284.35339 MR 2982744

[11] M. Hillairet, On the homogenization of the Stokes problem in a perforated domain. Arch. Ration. Mech. Anal. 230 (2018), no. 3, 1179-1228 Zbl 1432.35018 MR 3851058

[12] M. Hillairet and D. Wu, Effective viscosity of a polydispersed suspension. J. Math. Pures Appl. (9) 138 (2020), 413-447 Zbl 1441.35197 MR 4098775

[13] V. V. Jikov, S. M. Kozlov, and O. A. Oleĭnik, Homogenization of differential operators and integral functionals. Springer, Berlin, 1994 Zbl 0838.35001 MR 1329546

[14] T. Lévy and E. Sánchez-Palencia, Einstein-like approximation for homogenization with small concentration. II. Navier-Stokes equation. Nonlinear Anal. 9 (1985), no. 11, 1255-1268 Zbl 0581.76097 MR 813657

[15] B. Niethammer and R. Schubert, A local version of Einstein's formula for the effective viscosity of suspensions. SIAM J. Math. Anal. 52 (2020), no. 3, 2561-2591 Zbl 07245266 MR 4102716

[16] E. Sánchez-Palencia, Einstein-like approximation for homogenization with small concentration. I. Elliptic problems. Nonlinear Anal. 9 (1985), no. 11, 1243-1254 Zbl 0538.76086 MR 813656

[17] S. Serfaty, Coulomb gases and Ginzburg-Landau vortices. Zurich Lect. Adv. Math., European Mathematical Society (EMS), Zürich, 2015 Zbl 1335.82002 MR 3309890

[18] L. J. Walpole, The elastic behaviour of a suspension of spherical particles. Quart. J. Mech. Appl. Math. 25 (1972), 153-160 Zbl 0266.73007

[19] M. Zuzovsky, P. M. Adler, and H. Brenner, Spatially periodic suspensions of convex particles in linear shear flows. III. Dilute arrays of spheres suspended in Newtonian fluids. Phys. Fluids 26 (1983), 1714-1723 Zbl 0622.76114 
Received 18 June 2020; revised 1 March 2021; accepted 22 April 2021.

\section{David Gérard-Varet}

Institut de Mathématiques de Jussieu-Paris Rive Gauche (UMR 7586), Université de Paris, 75205 Paris, France; david.gerard-varet@imj-prg.fr

\section{Amina Mecherbet}

Institut de Mathématiques de Jussieu-Paris Rive Gauche (UMR 7586), Université de Paris, 75205 Paris, France; mecherbet@imj-prg.fr 